\title{
Visible-Light Photocatalytic Difluoroalkylation-Induced 1, 2-Heteroarene Migration of Allylic Alcohols in Batch and Flow
}

\author{
Xiao-Jing Wei and Timothy Noël*1(0) \\ Department of Chemical Engineering and Chemistry, Micro Flow Chemistry \& Process Technology, Eindhoven University of \\ Technology, Den Dolech 2, 5612 AZ Eindhoven, The Netherlands
}

\section{Supporting Information}

ABSTRACT: A convenient method for the preparation of $\mathrm{sp}^{3}$-rich heterocycles is reported. The method comprises a photocatalytic difluoroalkylation-induced 1,2-heteroarene migration of allylic alcohols. Here we describe for the first time the benefits of using flow to facilitate such migration reactions, including shorter reaction times, higher selectivities, and opportunities to scale the chemistry.

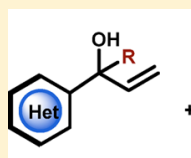

Starting Material prepared in flow

15 examples
$\mathrm{H}$ eteroarenes are widespread in pharmaceuticals, agrochemicals, and other bioactive molecules. Hence, the functionalization of heteroarenes remains a contemporary goal within synthetic organic chemistry. ${ }^{1}$ In recent years, there is a trend in medicinal chemistry to prepare more $\mathrm{sp}^{3}$-rich fragments (so-called "escape-from-flatland strategy") to reduce the attrition rate in drug discovery. ${ }^{2}$ To achieve this, the $\mathrm{sp}^{3}$ character of heteroarenes can be enhanced through alkylation via e.g. radical intermediates. ${ }^{3,4}$

An intriguing approach to access synthetically useful $\mathrm{sp}^{3}$-rich heteroarenes is the difunctionalization of alkenes initiated by a radical addition followed by a heteroaryl migration. ${ }^{5,6}$ Herein, we describe a novel 1,2-heteroarene migration induced by a photocatalytic radical difluoroalkylation. To prepare the target compounds, we developed a two-step protocol which starts from the corresponding heteroaryl ketones and includes a Grignard reaction and subsequent difluoroalkyl radical-induced migration reaction. As shown in this work, both reactions benefited substantially from continuous-flow processing.

Allylic alcohols are typically synthesized via a Grignard reaction between a heteroaryl ketone and vinyl magnesium halide. The reaction is exothermic in nature and requires strict cooling to avoid thermal runaway. Here, we have developed a continuous-flow protocol which allowed us to simultaneously handle the exotherm safely and prepare sufficient starting material for the subsequent photocatalytic migration reaction. The heteroaryl ketone was merged with vinylmagnesium bromide in a T-mixer and subsequently introduced in a capillary microreactor (ID $1.65 \mathrm{~mm} ; 700 \mu \mathrm{L}$ ). To avoid microreactor clogging, the mixer and microreactor were submerged into a sonicated icebath. ${ }^{8}$ A broad variety of heteroaryl allylic alcohols could be prepared using a residence time of only $5 \mathrm{~min}$ on a $5-10 \mathrm{mmol}$ scale as shown in Scheme 1; this includes 4-pyridinyl, 3-pyridinyl, 2-pyridinyl, pyrazinyl, benzothiophenyl, benzofuranyl, and thiophenyl bearing allylic alcohols. Notably, the reaction could be carried out at a higher temperature in flow $\left(0{ }^{\circ} \mathrm{C}\right.$ vs $-78{ }^{\circ} \mathrm{C}$ in batch $)$ which resulted in a reduced reaction time ( $5 \mathrm{~min}$ vs $2 \mathrm{~h}$ in batch). ${ }^{9}$

With a diverse set of allylic alcohols in hand, we commenced our investigations toward a broadly applicable difluoroalkylation-induced 1,2-heteroarene migration with 2-(pyridin-4yl)but-3-en-2-ol $\mathbf{2 a}$ as the benchmark substrate. Using ethyl bromodifluoroacetate $3 \mathrm{a}$ and $\mathrm{Ru}(\mathrm{bpy})_{3} \mathrm{Cl}_{2} \cdot 6 \mathrm{H}_{2} \mathrm{O}$ as the photocatalyst in the presence of a nitrogen base (Table 1 , entries 1-3), the target product could be obtained in encouraging yields (15-55\%) when the reaction mixture was subjected to blue irradiation. Switching to $f a c-\operatorname{Ir}(\text { ppy })_{3}$ as the photocatalyst improved the yield further to $67 \%$ (Table 1, entry 4). Screening other soluble bases revealed that optimal results could be obtained with imidazole (Table 1, entry 6). Several control experiments demonstrate the need for a base, photocatalyst, and light (Table 1, entries 7-9).

Having established the optimal reaction conditions, we set out to examine the substrate scope of the developed transformation (Scheme 2). 4-Pyridine-substituted allylic alcohols bearing various R-groups were subjected to the reaction conditions resulting in the targeted compounds in good yields $(\mathrm{R}=\mathrm{Me}, \mathrm{Et}, \mathrm{Ph}, \mathbf{2 a}-\mathrm{c})$. Interestingly, performing the reaction in flow resulted in a substantial reduction $(10 \mathrm{~min}$ in flow vs $6 \mathrm{~h}$ in batch) in reaction time and an increase in yield. ${ }^{10,11}$ As a consequence of the reduced exposure to light, the reaction mixture was typically cleaner resulting in a more facile purification by column chromatography. Halides on the pyridine moiety were well tolerated providing opportunities for further functionalization via e.g. cross coupling (4d, $4 \mathbf{f}, \mathbf{4 g}$ ). Surprisingly, 3-substituted pyridine allylic alcohols $(4 \mathbf{e}-\mathbf{h})$ could also give the aimed product smoothly. However, the yield is probably lower as a result of their lower reactivity in radical processeses. ${ }^{12}$ 2-Substituted pyridine allylic alcohol (4i) and 2-pyrazine-substituted allylic alcohols $(4 \mathbf{j}-\mathbf{k})$ underwent

Received: June 28, 2018

Published: July 18, 2018 
Scheme 1. Scope of the Continuous-Flow Grignard Synthesis of Allylic Alcohols ${ }^{a}$
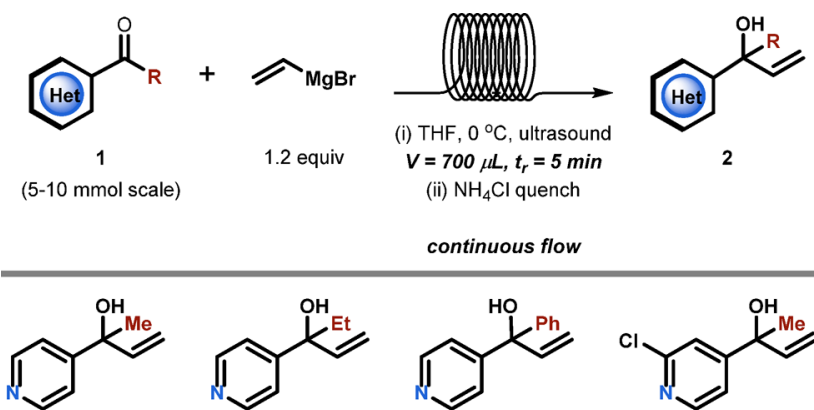

2a $(87 \%)^{b}$
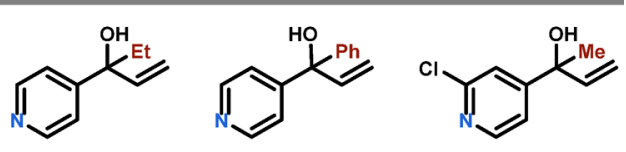

2c $(72 \%)$

2d (54\%)<smiles>C=CC(O)(O)c1cccnc1</smiles><smiles>C=CC(C)(O)c1ccc(Br)nc1</smiles><smiles>C=CC(C)(O)c1cncc(Br)c1</smiles>

$2 \mathrm{~g}(33 \%)$

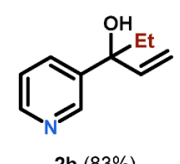<smiles>CCC(C)(O)c1ccccn1</smiles>

2i $(80 \%)$<smiles>C=CC(O)(O)c1cnccn1</smiles>

$2 \mathbf{j}(52 \%)^{c}$

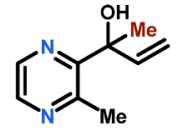

$2 k(61 \%)$

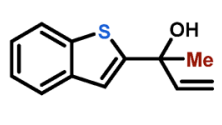

2I (85\%)

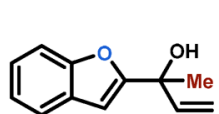

$2 \mathrm{~m}(77 \%)^{\circ}$

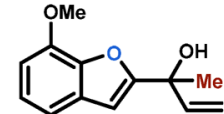

2n (80\%)

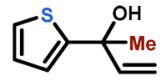

$20(35 \%)$
${ }^{a}$ Reaction conditions: Feed 1 contains $1(5.0 \mathrm{mmol})$ in $10 \mathrm{~mL}$ of THF; Feed 2 contains $10 \mathrm{~mL}$ of vinylmagnesium bromide $(1.67 \mathrm{M}$ in THF). Residence time of $5 \mathrm{~min}, 0{ }^{\circ} \mathrm{C}$, ultrasound. The reaction is quenched by saturated $\mathrm{NH}_{4} \mathrm{Cl}$ at the outlet of the reactor. Reported yields are those obtained after column chromatography. ${ }^{b}$ Carried out on a $10 \mathrm{mmol}$ scale. ${ }^{c}$ Residence time: $2.5 \mathrm{~min}$.

Table 1. Optimization of the Reaction Conditions ${ }^{a}$<smiles>C=CC(O)(O)c1ccncc1</smiles>

$2 a$<smiles>CCOC(=O)CC(C(C)=O)c1ccncc1</smiles>

Batch

$4 a$

\begin{tabular}{clll} 
entry & \multicolumn{1}{c}{ base } & \multicolumn{1}{c}{ photocatalyst } & yield $^{b}$ \\
\hline 1 & $\mathrm{NEt}_{3}$ & $\mathrm{Ru}(\mathrm{bpy})_{3} \mathrm{Cl}_{2} \cdot 6 \mathrm{H}_{2} \mathrm{O}$ & $31 \%$ \\
2 & $i \operatorname{Pr}_{2} \mathrm{NEt}$ & $\mathrm{Ru}(\mathrm{bpy})_{3} \mathrm{Cl}_{2} \cdot 6 \mathrm{H}_{2} \mathrm{O}$ & $15 \%$ \\
3 & $\mathrm{TMEDA}$ & $\mathrm{Ru}(\mathrm{bpy})_{3} \mathrm{Cl}_{2} \cdot 6 \mathrm{H}_{2} \mathrm{O}$ & $55 \%$ \\
4 & $\mathrm{TMEDA}$ & $\mathrm{fac}-\mathrm{Ir}(\mathrm{ppy})_{3}$ & $67 \%$ \\
5 & $\mathrm{DBU}$ & $f a c-\operatorname{Ir}(\mathrm{ppy})_{3}$ & $45 \%$ \\
6 & imidazole & $f a c-\operatorname{Ir}(\mathrm{ppy})_{3}$ & $\mathbf{8 3} \%$ \\
7 & - & $f a c-\operatorname{Ir}(\mathrm{ppy})_{3}$ & $57 \%$ \\
$8^{c}$ & imidazole & - & N.D. \\
$9^{d}$ & imidazole & $f a c-\operatorname{Ir}(\mathrm{ppy})_{3}$ & N.D.
\end{tabular}

${ }^{a}$ Reaction conditions: $2 \mathrm{a}(0.2 \mathrm{mmol}, 1$ equiv $), 3 a(0.6 \mathrm{mmol}, 3$ equiv), base (0.4 mmol, 2 equiv), photocatalyst (1 $\mathrm{mol} \%)$, dichloromethane $(1.0 \mathrm{~mL}), 12 \mathrm{~W}$ blue LEDs $(\lambda=450 \mathrm{~nm})$, room temperature, 6 h. ${ }^{b}$ Isolated yield. ${ }^{c}$ No photocatalyst. ${ }^{d}$ No light.

efficient migration under these photocatalytic conditions. Benzothiophene (41) migrates smoothly as well under our reaction conditions. However, other electron-rich heterocycles, such as benzofuran $(\mathbf{4 m - n )}$ and thiophene $(\mathbf{4 o})$, are
Scheme 2. Substrate Scope of the Photocatalytic RadicalInduced Heterocycle Migration-Variation of the Allylic Alcohol Substrate ${ }^{a}$

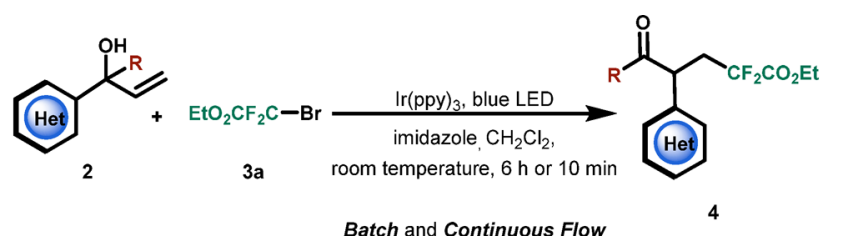<smiles>CCOC(=O)CC(CC(C(=O)OCC)c1ccncc1)c1ccncc1</smiles>
$\begin{array}{ccc}\text { 4a } & \text { 4b } & \text { 4c } \\ \text { Batch: } 83 \% & \text { Batch: } 89 \% & \text { Batch: } 82 \% \\ \text { Flow: } 91 \% & \text { Flow: } 97 \% & \text { Flow: } 98 \%\end{array}$<smiles>CCOC(=O)CC(C(C)=O)c1ccnc(Cl)c1</smiles><smiles>CCOC(=O)CC(C(C)=O)c1cccnc1</smiles><smiles>CCOC(=O)CC(CC(C)=O)c1ccc(Br)nc1</smiles>
4d
Batch: $87 \%$ Flow: $93 \%$ b<smiles>CCOC(=O)CC(C(C)=O)c1cncc(Br)c1</smiles>

Batch: $59 \%$

Flow: $66 \%$<smiles>CCOC(=O)CC(C(C)=O)c1cnccn1</smiles>

$4 \mathrm{j}$ Batch: $78 \%$ Flow: $91 \%$
$4 e$
Batch: $45 \%$<smiles>CCCCCCCCC(=O)C(CC(=O)OCC)C(CC(=O)OCC)c1ccccn1</smiles>

4h

Flow: $61 \%^{\circ}$<smiles>CCOC(=O)CCC(C(C)=O)c1nccnc1C</smiles>

4k

Batch: $83 \%$
Batch: $57 \%$
$4 i$ Batch: $62 \%$

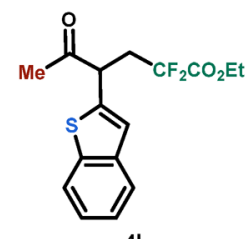

41

Batch: $60 \%$

Flow: $84 \%$<smiles>CCOC(=O)CCC(C(C)=O)c1cc2cccc(C(=O)OCC)c2o1</smiles>

${ }^{a}$ Reaction conditions in batch: $2 \mathrm{a}(0.2 \mathrm{mmol}), 3 \mathrm{a}(0.6 \mathrm{mmol})$, imidazole (0.4 mmol), $\mathrm{Ir}(\mathrm{ppy})_{3}(1 \mathrm{~mol} \%), \mathrm{CH}_{2} \mathrm{Cl}_{2}(1.0 \mathrm{~mL}), 12 \mathrm{~W}$ blue LEDs $(\lambda=450 \mathrm{~nm})$, room temperature, $6 \mathrm{~h}$. Reaction conditions in flow: 2a $(0.5 \mathrm{mmol}), 3 \mathrm{a}(1.5 \mathrm{mmol})$, imidazole $(1.0 \mathrm{mmol})$, $\operatorname{Ir}(\mathrm{ppy})_{3}(1 \mathrm{~mol} \%), \mathrm{CH}_{2} \mathrm{Cl}_{2}(5.0 \mathrm{~mL}), 12 \mathrm{~W}$ blue LEDs $(\lambda=450$ $\mathrm{nm})$, room temperature, residence time: $10 \mathrm{~min}$. Reported yields are those obtained after column chromatography. ${ }^{b}$ Residence time: 15 min. ${ }^{c}$ Residence time: 20 min. ${ }^{d}$ Residence time: 5 min.

susceptible for a double radical attack yielding the corresponding bisfunctionalized compounds in good yield. Also other 
difluoroalkyl radicals were able to induce the heteroaryl migrations (Scheme 3) using an analogous reaction protocol

Scheme 3. Substrate Scope of the Photocatalytic RadicalInduced Heterocycle Migration-Variation of the Difluoroalkyl Radical Precursor ${ }^{a}$
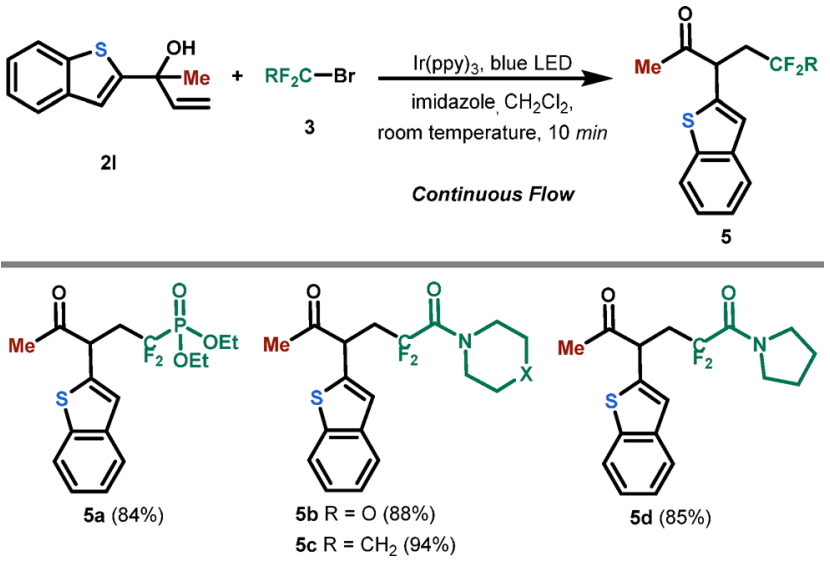

${ }^{a}$ Reaction conditions: 2a (1.0 mmol), 3a (3.0 mmol), $\operatorname{Ir}($ ppy)3 (1 mol \%), imidazole $(2.0 \mathrm{mmol}), \mathrm{CH}_{2} \mathrm{Cl}_{2}(1.0 \mathrm{~mL}), 12 \mathrm{~W}$ blue LEDs $(\lambda$ $=450 \mathrm{~nm}$ ), room temperature, residence time: $10 \mathrm{~min}$. Reported yields are those obtained after column chromatography.

where ethyl bromodifluoroacetate (3a) was replaced with bromodifluorophosphonate (yielding compound 5a), or various bromodifluoroacetamides (yielding compounds $\mathbf{5 b} \mathbf{b}$ d).

Based on the experimental data, a plausible mechanism is suggested in Figure 1. Upon light excitation, $f a c$ - $\left[\operatorname{Ir}(\mathrm{ppy})_{3}\right]^{*}$ can be oxidatively quenched by ethyl bromodifluoroacetate, generating the corresponding difluoroalkyl radical species. ${ }^{13}$ Indeed, radical trapping experiments with BHT (2,6-di-tertbutyl-4-methylphenol) showed that this species could be effectively captured (see Supporting Information). The radical subsequently adds to the olefin generating intermediate $\mathbf{A}$,

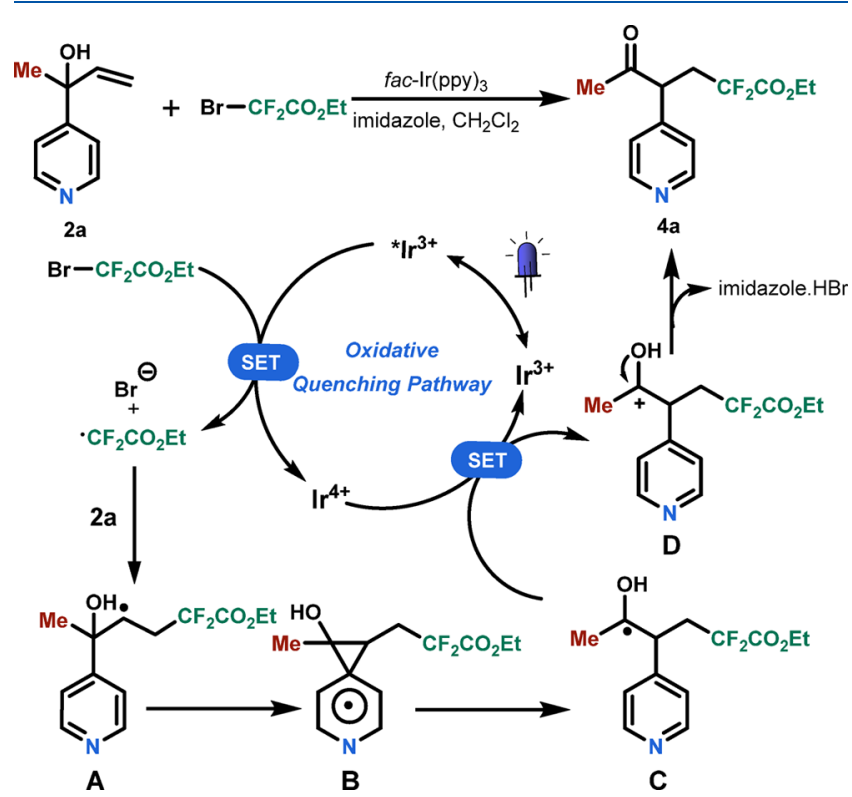

Figure 1. Proposed mechanism for the photocatalytic radical-induced heterocycle migration. which undergoes 1,2-heterocycle migration via a key spiro radical intermediate $\mathbf{B}$ to produce $\mathbf{C}$. Finally, the intermediate C was oxidized to obtain the aimed product $4 \mathbf{a}$.

In summary, we have developed a novel photocatalytic 1, 2heterocycle migration method which allows preparation of heterocycles with an $\mathrm{sp}^{3}$-enriched character. A variety of synthetically useful $\beta$-difluorinated $\alpha$-aryl heterocyclic ketones can be easily prepared under mild reaction conditions with excellent regioselectivity. The application of continuous flow allows a reduction in the reaction time (from $6 \mathrm{~h}$ to $10 \mathrm{~min}$ ) and provides higher reaction selectivity and potential for scaling the chemistry. Also interesting, the allylic alcohol substrates were prepared in flow via a classical Grignard reaction. The flow method enables safe handling of the reaction exotherm and allows preparation of sufficient quantities of starting material for the consecutive migration chemistry.

\section{EXPERIMENTAL SECTION}

All components as well as reagents and solvents were used as received without further purification, unless stated otherwise. Reagents and solvents were bought from Sigma-Aldrich, Acros Organics, Alfa Aesar, $\mathrm{ABCR}$, and TCI Chemicals. Photocatalyst fac-Ir(ppy) 3 (99\%) was bought from Sigma-Aldrich. Technical solvents were bought from VWR International and used as received. Product isolation was performed using silica (60, F254, Merck), and TLC analysis was performed using silica on aluminum foil TLC plates (F254, Supelco Sigma-Aldrich) with visualization under ultraviolet light (254 and 365 $\mathrm{nm})$ or appropriate TLC staining. NMR $\left({ }^{1} \mathrm{H},{ }^{13} \mathrm{C}\right.$, and $\left.{ }^{19} \mathrm{~F}\right)$ analyses were performed on a Bruker-Avance $400(400 \mathrm{MHz})$ in solvent $\mathrm{CDCl}_{3} .{ }^{1} \mathrm{H}$ NMR spectra are reported in parts per million (ppm) downfield relative to $\mathrm{CDCl}_{3}(7.27 \mathrm{ppm})$. All ${ }^{13} \mathrm{C}$ NMR spectra are reported in ppm relative to $\mathrm{CDCl}_{3}(77.00 \mathrm{ppm})$. NMR spectra uses the following abbreviations to describe the multiplicity: $s=$ singlet, $d$ = doublet, $\mathrm{t}=$ triplet, $\mathrm{q}=$ quartet, $\mathrm{m}=$ multiplet, $\mathrm{dd}=$ double doublet, $\mathrm{td}=$ triple doublet, $\mathrm{dt}=$ double triplet, $\mathrm{dq}=$ doublet of quartets, $\mathrm{brs}=$ broad singlet, dddd = doublet of doublet of doublet of doublets, ddd $=$ doublet of doublet of doublets, $\mathrm{dtd}=$ doublet of triplet of doublets. Mass spectra were recorded on a Finnigan MAT 4200S, a Bruker Daltonics Micro TOF, and a Waters Micromass Quatro LCZ (ESI); peaks are given in $\mathrm{m} / \mathrm{z}$ (\% of basis peak). The batch and flow reactions were carried out using 123-LEDs, stripe blue $2.5 \mathrm{~m}, 12 \mathrm{~W}$. The strips were wrapped on the inside of a 3D printed beaker. ${ }^{14}$

General Procedure for the Preparation of the 2-Heterocycle-but-3-en-2-ol Substrates in Flow. Heterocyclic ketone (5.0 mmol, 1.0 equiv) was dissolved in $10 \mathrm{~mL}$ of THF, which was subsequently degassed 3 times (freeze-pump-thaw: cooled to -78 ${ }^{\circ} \mathrm{C}$ and degassed via vacuum evacuation ( $5 \mathrm{~min}$ ), backfilled with argon, and warmed to room temperature) and taken up in a first syringe. Next, $6 \mathrm{~mL}$ of vinylmagnesium bromide ( $1 \mathrm{M}$ in THF) was dissolved in $4 \mathrm{~mL}$ of THF and taken up in a second syringe. These two syringes $(10 \mathrm{~mL})$ were mounted onto a single syringe pump. The reaction mixture was pumped through the PFA capillary microreactor (ID $=1.65 \mathrm{~mm}, 65 \mathrm{~cm}$ ), which was submerged in an ice water bath, which was sonicated to prevent microreactor clogging. The flow rate is $0.14 \mathrm{~mL} / \mathrm{min}$, which corresponds to a residence time of $5 \mathrm{~min}$. The quenching solvent is saturated $\mathrm{NH}_{4} \mathrm{Cl}$ water solution (Flow rate = $0.21 \mathrm{~mL} / \mathrm{min}$ ). The quenched solution was collected at the end of the reactor and was subsequently extracted by diethyl ether $(3 \times 30 \mathrm{~mL})$. The combined organic layers were washed with brine and dried with $\mathrm{MgSO}_{4}$. The product was purified via flash column chromatography using DCM/Acetone or cyclehexane/EtOAc as eluent.

2-(Pyridin-4-yl)but-3-en-2-ol (2a). The flow experiment was carried out on a $10.0 \mathrm{mmol}$ scale; $1.3 \mathrm{~g}$ of product was isolated as a white solid ( $87 \%$ yield). Purification: Column chromatography $\left(\mathrm{CH}_{2} \mathrm{Cl}_{2}\right.$ /acetone $\left.=30: 1\right) . \mathrm{Mp}=88-92{ }^{\circ} \mathrm{C} .{ }^{1} \mathrm{H}$ NMR $(400 \mathrm{MHz}$, $\left.\mathrm{CDCl}_{3}\right): \delta 8.57(\mathrm{~d}, J=4.0 \mathrm{~Hz}, 2 \mathrm{H}), 7.41(\mathrm{~d}, J=4.0 \mathrm{~Hz}, 2 \mathrm{H}), 6.17-$ $6.10(\mathrm{~m}, 1 \mathrm{H}), 5.33(\mathrm{~d}, J=16.0,1 \mathrm{H}), 5.22(\mathrm{~d}, J=12.0,1 \mathrm{H}), 2.36$ (brs, 
$1 \mathrm{H}), 1.65$ (s, 3H). ${ }^{13} \mathrm{C}$ NMR (100 MHz, $\left.\mathrm{CDCl}_{3}\right): \delta 155.6,149.5$, 149.4, 143.4, 120.4, 113.7, 74.1, 29.1. HRMS (ESI) $m / z:[\mathrm{M}+\mathrm{H}]^{+}$ calcd for $\mathrm{C}_{9} \mathrm{H}_{12} \mathrm{NO}, 150.0919$; found, 150.0921 .

3-(Pyridin-4-yl)pent-1-en-3-ol (2b). The flow experiment was carried out on a $5.0 \mathrm{mmol}$ scale; $749.8 \mathrm{mg}$ of product were isolated as a yellowish oil (92\% yield). Purification: Column chromatography $\left(\mathrm{CH}_{2} \mathrm{Cl}_{2} /\right.$ acetone $\left.=30: 1\right) .{ }^{1} \mathrm{H}$ NMR $\left(400 \mathrm{MHz}, \mathrm{CDCl}_{3}\right): \delta 8.49(\mathrm{~d}, J$ $=4.0 \mathrm{~Hz}, 2 \mathrm{H}), 7.37(\mathrm{~d}, J=4.0 \mathrm{~Hz}, 2 \mathrm{H}), 6.13(\mathrm{dd}, J=16.0,8.0 \mathrm{~Hz}$, $2 \mathrm{H}), 5.32$ (d, $J=16.0 \mathrm{~Hz}, 1 \mathrm{H}), 5.20(\mathrm{~d}, J=12.0 \mathrm{~Hz}, 1 \mathrm{H}), 2.36$ (brs, $1 \mathrm{H}), 1.92-1.87(\mathrm{~m}, 2 \mathrm{H}), 0.83(\mathrm{t}, J=8.0 \mathrm{~Hz}, 3 \mathrm{H}) .{ }^{13} \mathrm{C}$ NMR $(100$ $\left.\mathrm{MHz}, \mathrm{CDCl}_{3}\right): \delta 155.0,150.7,149.2,143.0,120.8,113.8,34.4,7.6$. HRMS (ESI) $m / z$ : $[\mathrm{M}+\mathrm{H}]^{+}$calcd for $\mathrm{C}_{10} \mathrm{H}_{14} \mathrm{NO}, 164.1075$; found, 164.1074.

1-Phenyl-1-(pyridin-4-yl)prop-2-en-1-ol (2c). The flow experiment was carried out on a $5.0 \mathrm{mmol}$ scale; $759.5 \mathrm{mg}$ of product were isolated as a white solid ( $72 \%$ yield). Purification: Column chromatography $\left(\mathrm{CH}_{2} \mathrm{Cl}_{2}\right.$ /acetone $\left.=16: 1\right) . \mathrm{Mp}=134-136{ }^{\circ} \mathrm{C} .{ }^{1} \mathrm{H}$ NMR $\left(400 \mathrm{MHz}, \mathrm{CDCl}_{3}\right): \delta 8.49(\mathrm{t}, J=4.0 \mathrm{~Hz}, 2 \mathrm{H}), 7.36-7.34(\mathrm{~m}$, $7 \mathrm{H}), 6.51-6.44(\mathrm{~m}, 1 \mathrm{H}), 5.41-5.34(\mathrm{~m}, 2 \mathrm{H}), 3.10$ (brs, $1 \mathrm{H}) .{ }^{13} \mathrm{C}$ NMR $\left(100 \mathrm{MHz}, \mathrm{CDCl}_{3}\right): \delta 155.0,149.1,144.5,142.1,128.5,127.9$, 126.9, 121.8, 115.5, 78.6. HRMS (ESI) $\mathrm{m} / z$ : $[\mathrm{M}+\mathrm{H}]^{+}$calcd for $\mathrm{C}_{14} \mathrm{H}_{14} \mathrm{NO}$, 212.1075; found, 212.1075 .

2-(2-Chloropyridin-4-yl)but-3-en-2-ol (2d). The flow experiment was carried out on a $5.0 \mathrm{mmol}$ scale; $494.1 \mathrm{mg}$ of product were isolated as a white solid ( $54 \%$ yield). Purification: Column chromatography $\left(\mathrm{CH}_{2} \mathrm{Cl}_{2} /\right.$ acetone $\left.=20: 1\right) . \mathrm{Mp}=54-56{ }^{\circ} \mathrm{C} .{ }^{1} \mathrm{H}$ NMR $\left(400 \mathrm{MHz}, \mathrm{CDCl}_{3}\right): \delta 8.15(\mathrm{~d}, J=4.0 \mathrm{~Hz}, 1 \mathrm{H}), 7.39-7.38(\mathrm{~m}$, $1 \mathrm{H}), 7.23(\mathrm{dd}, J=8.0,4.0 \mathrm{~Hz}, 1 \mathrm{H}), 6.00(\mathrm{~m}, 1 \mathrm{H}), 5.24(\mathrm{dd}, J=16.0$ $\mathrm{Hz}, 1 \mathrm{H}), 5.12(\mathrm{~d}, J=12.0 \mathrm{~Hz}, 1 \mathrm{H}), 3.64(\mathrm{~s}, 1 \mathrm{H}), 1.54(\mathrm{~s}, 3 \mathrm{H}) .{ }^{13} \mathrm{C}$ NMR $\left(100 \mathrm{MHz}, \mathrm{CDCl}_{3}\right): \delta 159.4,151.4,149.0,142.8,121.1,119.4$, 114.0, 73.8, 28.8. HRMS (ESI) $m / z:[\mathrm{M}+\mathrm{H}]^{+}$calcd for $\mathrm{C}_{9} \mathrm{H}_{11} \mathrm{ClNO}$, 184.0529; found, 184.0530 .

2-(Pyridin-3-yl)but-3-en-2-ol (2e). The flow experiment was carried out on a $5.0 \mathrm{mmol}$ scale; $662.8 \mathrm{mg}$ of product were isolated as a white solid ( $89 \%$ yield). Purification: Column chromatography $\left(\mathrm{CH}_{2} \mathrm{Cl}_{2}\right.$ /acetone $=8: 1$ to $\left.2: 1\right) . \mathrm{Mp}=27-29{ }^{\circ} \mathrm{C} .{ }^{1} \mathrm{H}$ NMR $(400$ $\left.\mathrm{MHz}, \mathrm{CDCl}_{3}\right): \delta 8.63(\mathrm{~s}, 1 \mathrm{H}), 8.41-8.39(\mathrm{~m}, 1 \mathrm{H}), 7.76(\mathrm{dd}, J=16.0$, $4.0 \mathrm{~Hz}, 1 \mathrm{H}), 7.29-7.26(\mathrm{~m}, 1 \mathrm{H}), 6.20-6.13(\mathrm{~m}, 1 \mathrm{H}), 5.32(\mathrm{~d}, J=$ $16.0 \mathrm{~Hz}, 1 \mathrm{H}), 5.20(\mathrm{~d}, J=12.0 \mathrm{~Hz}, 1 \mathrm{H}), 2.85($ brs, $1 \mathrm{H}), 1.68(\mathrm{~s}, 3 \mathrm{H})$. ${ }^{13} \mathrm{C}$ NMR $\left(100 \mathrm{MHz}, \mathrm{CDCl}_{3}\right): \delta 147.8,147.0,144.1,144.0,142.0$, 133.4, 123.2, 113.4, 113.3, 73.5, 29.4. HRMS (ESI) $m / z:[\mathrm{M}+\mathrm{H}]^{+}$ calcd for $\mathrm{C}_{9} \mathrm{H}_{12} \mathrm{NO}, 150.0919$; found, 150.0918 .

2-(6-Bromopyridin-3-yl)but-3-en-2-ol (2f). The flow experiment was carried out on a $5.0 \mathrm{mmol}$ scale; $669.5 \mathrm{mg}$ of product were isolated as a yellow oil (59\% yield). Purification: Column chromatography $\left(\mathrm{CH}_{2} \mathrm{Cl}_{2}\right.$ /acetone $\left.=20: 1\right) .{ }^{1} \mathrm{H}$ NMR $(400 \mathrm{MHz}$, $\left.\mathrm{CDCl}_{3}\right): \delta 8.55(\mathrm{~s}, 1 \mathrm{H}), 8.50(\mathrm{~s}, 1 \mathrm{H}), 7.99-7.98(\mathrm{~m}, 1 \mathrm{H}), 6.15-6.08$ $(\mathrm{m}, 1 \mathrm{H}), 5.35-5.20(\mathrm{~m}, 2 \mathrm{H}), 3.21(\mathrm{brs}, 1 \mathrm{H}), 1.66(\mathrm{~s}, 3 \mathrm{H}) .{ }^{13} \mathrm{C}$ NMR $\left(100 \mathrm{MHz}, \mathrm{CDCl}_{3}\right): \delta 148.8,145.2,144.0,143.4,136.1,120.6,113.9$, 73.0, 29.3. HRMS (ESI) $\mathrm{m} / z$ : $[\mathrm{M}+\mathrm{H}]^{+}$calcd for $\mathrm{C}_{9} \mathrm{H}_{11} \mathrm{BrNO}$, 228.0024; found, 228.0025 .

2-(5-Bromopyridin-3-yl)but-3-en-2-ol (2g). The batch experiment was carried out on a $5.0 \mathrm{mmol}$ scale; $374.0 \mathrm{mg}$ of product were isolated as a brown oil (33\% yield). Purification: Column chromatography $(\mathrm{DCM} /$ Acetone $=20: 1) .{ }^{1} \mathrm{H}$ NMR $(400 \mathrm{MHz}$, $\left.\mathrm{CDCl}_{3}\right): \delta 8.44(\mathrm{~s}, 1 \mathrm{H}), 7.67(\mathrm{dd}, J=8.0,4.0 \mathrm{~Hz}, 1 \mathrm{H}), 7.44(J=8.0$, $1 \mathrm{H}), 6.12(\mathrm{dd}, J=16.0,8.0 \mathrm{~Hz}, 1 \mathrm{H}), 5.33-5.21(\mathrm{~m}, 2 \mathrm{H}), 2.18(\mathrm{~m}$, $1 \mathrm{H}), 1.66(\mathrm{~s}, 3 \mathrm{H}) .{ }^{13} \mathrm{C}$ NMR $\left(100 \mathrm{MHz}, \mathrm{CDCl}_{3}\right): \delta 147.7,143.4$, $141.2,140.5,136.1,127.5,113.9,73.3,29.4$. HRMS (ESI) $m / z:[\mathrm{M}+$ $\mathrm{H}]^{+}$calcd for $\mathrm{C}_{9} \mathrm{H}_{11} \mathrm{BrNO}, 228.0024$; found, 228.0025 .

3-(Pyridin-3-yl)pent-1-en-3-ol (2h). The flow experiment was carried out on a $5.0 \mathrm{mmol}$ scale (residence time: $10 \mathrm{~min}$ ); $676.4 \mathrm{mg}$ of product were isolated as a yellow oil ( $83 \%$ yield). Purification: Column chromatography $\left(\mathrm{CH}_{2} \mathrm{Cl}_{2}\right.$ /acetone $=8: 1$ to $\left.2: 1\right) .{ }^{1} \mathrm{H}$ NMR $\left(400 \mathrm{MHz}, \mathrm{CDCl}_{3}\right): \delta 8.62(\mathrm{~s}, 1 \mathrm{H}), 8.42-8.39(\mathrm{~m}, 1 \mathrm{H}), 7.78(\mathrm{dd}, J=$ $8.0,4.0 \mathrm{~Hz}, 1 \mathrm{H}), 7.26-7.22(\mathrm{~m}, 1 \mathrm{H}), 6.19-6.12(\mathrm{~m}, 1 \mathrm{H}), 5.32(\mathrm{~d}, J=$ $16.0 \mathrm{~Hz}, 1 \mathrm{H}), 5.20(\mathrm{~d}, J=8.0 \mathrm{~Hz}, 1 \mathrm{H}), 3.48(\mathrm{brs}, 1 \mathrm{H}), 1.96-1.89(\mathrm{~m}$, $2 \mathrm{H}), 0.84(\mathrm{t}, J=8.0 \mathrm{~Hz}, 3 \mathrm{H}) .{ }^{13} \mathrm{C}$ NMR $\left(100 \mathrm{MHz}, \mathrm{CDCl}_{3}\right): \delta 147.8$, 147.2, 143.3, 141.1, 133.7, 123.0, 113.5, 75.7, 34.6, 7.7. HRMS (ESI) $m / z:[\mathrm{M}+\mathrm{H}]^{+}$calcd for $\mathrm{C}_{10} \mathrm{H}_{14} \mathrm{NO}, 164.1075$; found, 164.1078 .
1-(Pyridin-2-yl)hexan-1-one (1i). The compound was made according to a literature procedure. ${ }^{15}{ }^{1} \mathrm{H}$ NMR $(400 \mathrm{MHz}$, $\left.\mathrm{CDCl}_{3}\right): \delta 8.68(\mathrm{~d}, J=4.0 \mathrm{~Hz}, 1 \mathrm{H}), 8.04(\mathrm{~d}, J=4.0 \mathrm{~Hz}, 1 \mathrm{H}), 7.82$ $(\mathrm{t}, J=8.0 \mathrm{~Hz}, 1 \mathrm{H}), 7.46(\mathrm{dd}, J=8.0,4.0 \mathrm{~Hz}, 1 \mathrm{H}), 3.22(\mathrm{t}, J=8.0 \mathrm{~Hz}$, $2 \mathrm{H}), 1.73(\mathrm{t}, J=8.0 \mathrm{~Hz}, 2 \mathrm{H}), 1.40-1.36(\mathrm{~m}, 4 \mathrm{H}), 0.92-0.88(\mathrm{~m}$, $3 \mathrm{H}) .{ }^{13} \mathrm{C}$ NMR $\left(100 \mathrm{MHz}, \mathrm{CDCl}_{3}\right): \delta 202.1,153.5,148.8,136.9$, 126.9, 121.8, 37.7, 31.5, 23.6, 22.5, 13.9.

3-(Pyridin-4-yl)oct-1-en-3-ol (2i). The flow experiment was carried out on a $5.0 \mathrm{mmol}$ scale; a $1.0 \mathrm{~g}$ mixture of product and starting material (5:1 based on HNMR) was obtained as a yellow oil $(80 \%$ yield). Purification: Column chromatography $\left(\mathrm{CH}_{2} \mathrm{Cl}_{2} /\right.$ acetone $=8: 1$ to $2: 1)$. ${ }^{1} \mathrm{H}$ NMR $\left(400 \mathrm{MHz}, \mathrm{CDCl}_{3}\right): \delta 8.55-8.52(\mathrm{~m}, 1 \mathrm{H}), 7.76-$ $7.73(\mathrm{~m}, 1 \mathrm{H}), 7.39-7.36(\mathrm{~m}, 1 \mathrm{H}), 7.26-7.23(\mathrm{~m}, 1 \mathrm{H}), 6.12(\mathrm{dd}, J=$ 16.0, $8.0 \mathrm{~Hz}, 1 \mathrm{H}), 5.42(\mathrm{~d}, J=16.0 \mathrm{~Hz}, 1 \mathrm{H}), 5.34(\mathrm{br} \mathrm{s}, 1 \mathrm{H}), 5.13(\mathrm{~d}$, $J=16.0 \mathrm{~Hz}, 1 \mathrm{H}), 1.92-1.87(\mathrm{~m}, 2 \mathrm{H}), 1.46-1.37(\mathrm{~m}, 2 \mathrm{H}), 1.28-1.20$ $(\mathrm{m}, 4 \mathrm{H}), 0.84(\mathrm{t}, J=4.0 \mathrm{~Hz}, 3 \mathrm{H}) .{ }^{13} \mathrm{C} \mathrm{NMR}\left(100 \mathrm{MHz}, \mathrm{CDCl}_{3}\right): \delta$ $162.5,148.9,143.0,136.8,126.9,122.3,121.7,120.5,113.8,76.3$, 41.2, 32.0, 22.9, 14.0. HRMS (ESI) $m / z:[\mathrm{M}+\mathrm{H}]^{+}$calcd for $\mathrm{C}_{13} \mathrm{H}_{20} \mathrm{NO}$ : 206.1545; found, 206.1542.

2-(Pyrazin-2-yl)but-3-en-2-ol (2j). The flow experiment was carried out on a $5.0 \mathrm{mmol}$ scale; $390.0 \mathrm{mg}$ of product were isolated as a colorless oil ( $52 \%$ yield). Purification: Column chromatography $\left(\mathrm{CH}_{2} \mathrm{Cl}_{2}\right.$ /acetone $\left.=20: 1\right) .{ }^{1} \mathrm{H} \mathrm{NMR}\left(400 \mathrm{MHz}, \mathrm{CDCl}_{3}\right): \delta 8.77(\mathrm{~s}$, $1 \mathrm{H}), 8.51-8.50(\mathrm{~m}, 2 \mathrm{H}), 6.20-6.12(\mathrm{~m}, 1 \mathrm{H}), 5.42-5.38(\mathrm{~m}, 1 \mathrm{H})$, 5.21-5.18 (m, $1 \mathrm{H}), 3.95$ (brs, $1 \mathrm{H}), 1.69(\mathrm{~s}, 3 \mathrm{H}) .{ }^{13} \mathrm{C}$ NMR $(100$ $\left.\mathrm{MHz}, \mathrm{CDCl}_{3}\right): \delta 159.3,143.0,142.9,142.4,142.3,113.8,73.7,28.1$. HRMS (ESI) $m / z:[\mathrm{M}+\mathrm{H}]^{+}$calcd for $\mathrm{C}_{8} \mathrm{H}_{11} \mathrm{~N}_{2} \mathrm{O}, 151.0871$; found, 151.0872 .

2-(3-Methylpyrazin-2-yl)but-3-en-2-ol (2k). The flow experiment was carried out on a $5.0 \mathrm{mmol}$ scale; $500.0 \mathrm{mg}$ of product were isolated as a colorless oil (61\% yield). Purification: Column chromatography $\left(\mathrm{CH}_{2} \mathrm{Cl}_{2}\right.$ /acetone $\left.=30: 1\right) .{ }^{1} \mathrm{H} \mathrm{NMR}(400 \mathrm{MHz}$, $\left.\mathrm{CDCl}_{3}\right): \delta 8.41-8.30(\mathrm{~m}, 2 \mathrm{H}), 6.09-6.01(\mathrm{~m}, 1 \mathrm{H}), 5.66(\mathrm{brs}, 1 \mathrm{H})$, 5.36-5.20 (m, 2H), 2.65-2.64 (m, 3H), $1.67(\mathrm{~s}, 3 \mathrm{H}) .{ }^{13} \mathrm{C}$ NMR $(100$ $\left.\mathrm{MHz}, \mathrm{CDCl}_{3}\right): \delta 155.9,152.1,142.5,141.3,138.8,114.7,73.3,25.7$, 25.5. HRMS (ESI) $m / z:[\mathrm{M}+\mathrm{H}]^{+}$calcd for $\mathrm{C}_{9} \mathrm{H}_{13} \mathrm{~N}_{2} \mathrm{O}, 165.1028$; found, 165.1029 .

2-(Benzo[b]thiophen-2-yl)but-3-en-2-ol (2l). The flow experiment was carried out on a $5.0 \mathrm{mmol}$ scale; $867.0 \mathrm{mg}$ of product were isolated as a transparent oil ( $85 \%$ yield). Purification: Column chromatography (Cyclohexane/EtOAc $=20: 1) .{ }^{1} \mathrm{H}$ NMR $(400 \mathrm{MHz}$, $\left.\mathrm{CDCl}_{3}\right): \delta 7.81-7.69(\mathrm{~m}, 2 \mathrm{H}), 7.35-7.29(\mathrm{~m}, 2 \mathrm{H}), 7.18(\mathrm{~s}, 1 \mathrm{H}), 6.27$ (dd, $J=17.2,10.6 \mathrm{~Hz}, 1 \mathrm{H}), 5.42(\mathrm{dd}, J=17.2,0.9 \mathrm{~Hz}, 1 \mathrm{H}), 5.22(\mathrm{dd}$, $J=10.6,0.9 \mathrm{~Hz}, 1 \mathrm{H}), 1.80(\mathrm{~s}, 3 \mathrm{H}) .{ }^{13} \mathrm{C} \mathrm{NMR}\left(100 \mathrm{MHz}^{\mathrm{C} C D C l}\right)_{3}: \delta$ $152.2,143.5,139.7,139.5,124.3,124.1,123.5,122.3,119.7,113.1$, 73.7, 30.0. HRMS (ESI) $\mathrm{m} / z$ : $[\mathrm{M}+\mathrm{H}]^{+}$calcd for $\mathrm{C}_{12} \mathrm{H}_{13} \mathrm{SO}$, 205.0687; found, 205.0686 .

2-(Benzofuran-2-yl)but-3-en-2-ol (2m). The flow experiment was carried out on a $5.0 \mathrm{mmol} \mathrm{scale;} 723.8 \mathrm{mg}$ of product were isolated as a colorless oil (77\% yield). Purification: Column chromatography (Cyclohexane/EtOAc $=4: 1) .{ }^{1} \mathrm{H}$ NMR $\left(400 \mathrm{MHz}, \mathrm{CDCl}_{3}\right): \delta 7.56-$ $7.46(\mathrm{~m}, 2 \mathrm{H}), 7.28-7.22(\mathrm{~m}, 2 \mathrm{H}), 6.63(\mathrm{~s}, 1 \mathrm{H}), 6.25(\mathrm{dd}, J=17.3$, $10.6 \mathrm{~Hz}, 1 \mathrm{H}), 5.41(\mathrm{dd}, J=17.3,0.8 \mathrm{~Hz}, 1 \mathrm{H}), 5.25(\mathrm{dd}, J=10.6,0.9$ $\mathrm{Hz}, 1 \mathrm{H}), 2.38$ (brs, $1 \mathrm{H}), 1.76(\mathrm{~s}, 3 \mathrm{H}) .{ }^{13} \mathrm{C} \mathrm{NMR}\left(100 \mathrm{MHz}, \mathrm{CDCl}_{3}\right)$ : $\delta 161.0,145.3,144.0,141.7,129.8,123.4,113.8,113.4,106.3,102.2$, 71.8, 55.9, 26.9. HRMS (ESI) $m / z:[\mathrm{M}+\mathrm{H}]^{+}$calcd for $\mathrm{C}_{12} \mathrm{H}_{13} \mathrm{O}_{2}$, 189.0916; found, 189.0916 .

2-(7-Methoxybenzofuran-2-yl)but-3-en-2-ol (2n). The flow experiment was carried out on a $5.0 \mathrm{mmol}$ scale; $870 \mathrm{mg}$ of product were isolated as a colorless oil ( $80 \%$ yield). Purification: Column chromatography (Cyclohexane/EtOAc $=4: 1) .{ }^{1} \mathrm{H}$ NMR $(400 \mathrm{MHz}$, $\left.\mathrm{CDCl}_{3}\right): \delta 7.15-7.14(\mathrm{~m}, 2 \mathrm{H}), 6.79(\mathrm{dd}, J=5.1,4.0 \mathrm{~Hz}, 1 \mathrm{H}), 6.62(\mathrm{~s}$, $1 \mathrm{H}), 6.26(\mathrm{dd}, J=16.0,8.0 \mathrm{~Hz}, 1 \mathrm{H}), 5.40(\mathrm{~d}, J=20.0 \mathrm{~Hz}, 1 \mathrm{H}), 5.23$ $(\mathrm{d}, J=8.0 \mathrm{~Hz}, 1 \mathrm{H})), 4.00(\mathrm{~s}, 3 \mathrm{H}), 2.50($ brs, $1 \mathrm{H}), 1.76(\mathrm{~s}, 3 \mathrm{H}) \cdot{ }^{13} \mathrm{C}$ $\operatorname{NMR}\left(100 \mathrm{MHz}, \mathrm{CDCl}_{3}\right): \delta 161.0,145.3,144.0,141.7,129.8,123.4$, $113.8,113.4,106.3,102.2,71.8,55.9,26.9$. HRMS (ESI) $m / z:[\mathrm{M}+$ $\mathrm{H}]^{+}$calcd for $\mathrm{C}_{13} \mathrm{H}_{15} \mathrm{O}_{3}, 219.1021$; found, 219.1021.

2-(Thiophen-2-yl)but-3-en-2-ol (2o). The flow experiment was carried out on a $5.0 \mathrm{mmol} \mathrm{scale;} 270.0 \mathrm{mg}$ of product were isolated as a colorless oil (35\% yield). Purification: Column chromatography 
$($ Cyclohexane/EtOAc $=4: 1) .{ }^{1} \mathrm{H}$ NMR $\left(400 \mathrm{MHz}, \mathrm{CDCl}_{3}\right): \delta 7.24$ $(\mathrm{dd}, J=4.0,2.3 \mathrm{~Hz}, 2 \mathrm{H}), 6.97-6.96(\mathrm{~m}, 2 \mathrm{H}), 6.23(\mathrm{~d}, J=16.0,8.0$ $\mathrm{Hz}, 1 \mathrm{H}), 5.40-5.16(\mathrm{~m}, 2 \mathrm{H}), 2.18$ (brs, $1 \mathrm{H}), 1.75(\mathrm{~s}, 3 \mathrm{H}) .{ }^{13} \mathrm{C}$ NMR $\left(100 \mathrm{MHz}, \mathrm{CDCl}_{3}\right): \delta 151.6,144.0,126.7,124.6,123.2,112.5,73.3$, 30.2. HRMS (ESI) $m / z:[\mathrm{M}+\mathrm{H}]^{+}$calcd for $\mathrm{C}_{8} \mathrm{H}_{11} \mathrm{OS}, 155.0531$; found, 155.0529 .

General Procedure for the Photocatalytic 1,2-Heterocycle Migration in Batch. An oven-dried reaction tube $(7.5 \mathrm{~mL})$ was charged with 2-heterocycle-but-3-en-2-ol 2 ( $0.2 \mathrm{mmol}, 1.0$ equiv), ethyl bromodifluoroacetate $\left(0.6 \mathrm{mmol}, 3.0\right.$ equiv), $f a c-\operatorname{Ir}(\mathrm{ppy})_{3}(1.3$ $\mathrm{mg}, 1.0 \mathrm{~mol} \%)$, imidazole $(27.3 \mathrm{mg}, 0.4 \mathrm{mmol}, 2$ equiv), and a magnetic stirring bar in dichloromethane $(1.0 \mathrm{~mL})$, sealed with a rubber septum, and subsequently degassed 3 times (freeze-pumpthaw: cooled to $-78{ }^{\circ} \mathrm{C}$ and degassed via vacuum evacuation $(5 \mathrm{~min})$, backfilled with argon, and warmed to room temperature). Next the reaction mixture was irradiated with blue LEDs (at approximately 1 $\mathrm{cm}$ distance from the light source). The temperature in the reactor was kept at room temperature using pressurized air. After $6 \mathrm{~h}$, the reaction mixture was transferred to a $50 \mathrm{~mL}$ flask with about $20 \mathrm{~mL}$ of $\mathrm{CH}_{2} \mathrm{Cl}_{2}$. The solvent was subsequently removed under reduced pressure, and the residue was purified by silica gel column chromatography using dichloromethane/acetone to give the desired product.

General Procedure for the Photocatalytic 1,2-Heterocycle Migration in Flow. 2-Heterocycle-but-3-en-2-ol 2 (1.0 mmol, 1.0 equiv), ethyl bromodifluoroacetate ( $3.0 \mathrm{mmol}, 3.0$ equiv), fac$\operatorname{Ir}(\text { ppy })_{3}(6.5 \mathrm{mg}, 1.0 \mathrm{~mol} \%)$, imidazole $(136.2 \mathrm{mg}, 2.0 \mathrm{mmol}, 2$ equiv), and a magnetic stirring bar in dichloromethane $(10.0 \mathrm{~mL})$ were combined and subsequently degassed 3 times (freeze-pumpthaw: cooled to $-78{ }^{\circ} \mathrm{C}$ and degassed via vacuum evacuation $(5 \mathrm{~min})$, backfilled with argon, and warmed to room temperature). This reaction mixture was then transferred into a syringe $(10 \mathrm{~mL})$ and mounted onto a syringe pump. The reaction mixture was pumped through the microreactor with the desired flow rate $(0.053 \mathrm{~mL}$ $\mathrm{min}^{-1}$ ). The microreactor assembly was irradiated with a blue LED array $(1.5 \times 3.12 \mathrm{~W})$ at room temperature. The continuous reaction was allowed to reach steady state prior to collection of the product fractions. A standard residence time of $10 \mathrm{~min}$ was utilized. The crude product was collected at the end of the reactor. Workup and purification were done following the batch procedure.

Ethyl 2,2-Difluoro-5-oxo-4-(pyridin-4-yl)hexanoate (4a). The batch reaction was carried out on a $0.2 \mathrm{mmol}$ scale, and the flow experiment was carried out on a $0.5 \mathrm{mmol}$ scale $(2.5 \mathrm{~mL}$ liquid was taken); $44.9 \mathrm{mg}$ and $61.7 \mathrm{mg}$ were obtained in batch and flow (83\%, $91 \%$ yields). The product was a yellow oil. Purification: Column chromatography $\left(\mathrm{CH}_{2} \mathrm{Cl}_{2} /\right.$ Acetone $\left.=30: 1\right) .{ }^{1} \mathrm{H}$ NMR $(400 \mathrm{MHz}$, $\left.\mathrm{CDCl}_{3}\right): \delta 8.53(\mathrm{t}, J=4.0 \mathrm{~Hz}, 2 \mathrm{H}), 7.11-7.09(\mathrm{~m}, 2 \mathrm{H}), 4.13(\mathrm{dq}, J=$ 8.0, $4.0 \mathrm{~Hz}, 2 \mathrm{H}), 3.97(\mathrm{dd}, J=8.0,4.0 \mathrm{~Hz}, 1 \mathrm{H}), 3.03(\mathrm{dtd}, J=17.2$, $15.3,5.6 \mathrm{~Hz}, 1 \mathrm{H}), 2.27(\mathrm{dtd}, J=17.2,15.3,5.6 \mathrm{~Hz}, 1 \mathrm{H}), 2.05(\mathrm{~s}, 3 \mathrm{H})$, $1.24(\mathrm{t}, J=8.0 \mathrm{~Hz}, 3 \mathrm{H}) .{ }^{13} \mathrm{C} \mathrm{NMR}\left(100 \mathrm{MHz}, \mathrm{CDCl}_{3}\right): \delta 203.9$, $163.5(\mathrm{t}, J=33.0 \mathrm{~Hz}), 150.6,146.1,123.3,114.8(\mathrm{t}, J=250.0 \mathrm{~Hz})$, $63.1,51.7(\mathrm{t}, J=3.0 \mathrm{~Hz}), 36.2(\mathrm{t}, J=23.0 \mathrm{~Hz}), 29.0,13.7 .{ }^{13} \mathrm{~F}$ NMR $\left(376 \mathrm{MHz} \mathrm{CDCl}_{3}\right): \delta-104.67$ to $-104.80(\mathrm{~m})$. HRMS (EI) $\mathrm{m} / z$ : [M $+\mathrm{H}]^{+}$calcd for $\mathrm{C}_{13} \mathrm{H}_{16} \mathrm{~F}_{2} \mathrm{NO}_{3}$, 272.1098; found, 272.1080.

Ethyl 2,2-Difluoro-5-oxo-4-(pyridin-4-yl)heptanoate (4b). The batch reaction was carried out on a $0.2 \mathrm{mmol}$ scale, and the flow experiment was carried out on a $0.5 \mathrm{mmol}$ scale $(3.2 \mathrm{~mL}$ liquid was taken); $50.7 \mathrm{mg}$ and $88.0 \mathrm{mg}$ of product were obtained in batch and flow as a pale yellow oil (89\%, 97\% yields). Purification: Column chromatography $\left(\mathrm{CH}_{2} \mathrm{Cl}_{2}\right.$ /Acetone $=30: 1$ to $\left.10: 1\right) .{ }^{1} \mathrm{H}$ NMR $(400$ $\left.\mathrm{MHz}, \mathrm{CDCl}_{3}\right): 8.60(\mathrm{~d}, J=4.0 \mathrm{~Hz}, 2 \mathrm{H}), 7.20(\mathrm{~d}, J=4.0 \mathrm{~Hz}, 2 \mathrm{H})$, $4.38-4.15(\mathrm{~m}, 2 \mathrm{H}), 4.06(\mathrm{dd}, J=7.7,5.3 \mathrm{~Hz}, 1 \mathrm{H}), 3.19-3.05(\mathrm{~m}$, $1 \mathrm{H}), 2.61-2.36(\mathrm{~m}, 2 \mathrm{H}), 2.40-2.20(\mathrm{~m}, 1 \mathrm{H}), 1.32(\mathrm{t}, J=8.0 \mathrm{~Hz}$, $3 \mathrm{H}), 1.00(\mathrm{t}, J=8.0 \mathrm{~Hz}, 3 \mathrm{H}) .{ }^{13} \mathrm{C}$ NMR $\left(100 \mathrm{MHz}, \mathrm{CDCl}_{3}\right): \delta 206.8$, $163.5(\mathrm{t}, J=32.0 \mathrm{~Hz}), 150.1,146.9,123.4,114.8(\mathrm{t}, J=250.0 \mathrm{~Hz})$, $63.2,50.8(\mathrm{t}, J=3.0 \mathrm{~Hz}), 36.6(\mathrm{t}, J=23.0 \mathrm{~Hz}), 35.3,13.8 .{ }^{19} \mathrm{~F}$ NMR $\left(376 \mathrm{MHz}, \mathrm{CDCl}_{3}\right.$ ): $\delta-104.79(\mathrm{~m})$. HRMS (ESI) $m / z:[\mathrm{M}+\mathrm{Na}]^{+}$ calcd for $\mathrm{C}_{14} \mathrm{H}_{17} \mathrm{~F}_{2} \mathrm{NO}_{3} \mathrm{Na}$, 308.1074; found, 308.1076.

Ethyl 2,2-Difluoro-5-oxo-5-phenyl-4-(pyridin-4-yl)pentanoate (4c). The batch reaction was carried out on a $0.2 \mathrm{mmol} s c a l e$, and the flow experiment was carried out on a $0.5 \mathrm{mmol}$ scale $(3.0 \mathrm{~mL}$ liquid was taken); $54.7 \mathrm{mg}$ and $97.7 \mathrm{mg}$ of product were obtained in batch and flow as a yellow oil (82\%, 98\% yields). Purification: Column chromatography $\left(\mathrm{CH}_{2} \mathrm{Cl}_{2} /\right.$ Acetone $\left.=20: 1\right) .{ }^{1} \mathrm{H}$ NMR $(400$ $\left.\mathrm{MHz}, \mathrm{CDCl}_{3}\right): \delta 8.55$ (brs, $\left.2 \mathrm{H}\right), 7.94(\mathrm{dd}, J=8.0,4.0 \mathrm{~Hz}, 2 \mathrm{H}), 7.55$ $(\mathrm{t}, J=8.0 \mathrm{~Hz}, 1 \mathrm{H}), 7.43(\mathrm{t}, J=8.0 \mathrm{~Hz}, 2 \mathrm{H}), 7.26(\mathrm{~d}, J=8.0 \mathrm{~Hz}, 2 \mathrm{H})$, $4.98(\mathrm{t}, J=8.0 \mathrm{~Hz}, 2 \mathrm{H}), 4.27-4.10(\mathrm{~m}, 2 \mathrm{H}), 3.28(\mathrm{dtd}, J=18.2,15.3$, $7.8 \mathrm{~Hz}), 2.52(\mathrm{dtd}, J=18.0,14.9,5.1 \mathrm{~Hz}), 1.26(\mathrm{t}, J=7.2 \mathrm{~Hz}, 3 \mathrm{H})$. ${ }^{13} \mathrm{C}$ NMR $\left(100 \mathrm{MHz}, \mathrm{CDCl}_{3}\right): \delta 196.1,163.5(\mathrm{t}, J=33.0 \mathrm{~Hz}), 150.2$, 147.1, 135.2, 133.8, 128.8, 128.7, 123.4, $114.9(\mathrm{t}, J=250.0 \mathrm{~Hz}), 63.1$, 46.0, $37.8(\mathrm{t}, J=23.0 \mathrm{~Hz}), 13.7 .{ }^{13} \mathrm{~F}$ NMR $\left(376 \mathrm{MHz} \mathrm{CDCl}_{3}\right): \delta$ -104.43 (ddd, $J=57.3,18.0,15.1 \mathrm{~Hz}$ ). HRMS (ESI) $\mathrm{m} / z$ : $[\mathrm{M}+$ $\mathrm{Na}]^{+}$calcd for $\mathrm{C}_{18} \mathrm{H}_{17} \mathrm{~F}_{2} \mathrm{NO}_{3} \mathrm{Na}, 356.1074$; found, 356.1078 .

Ethyl 4-(2-Chloropyridin-4-yl)-2,2-difluoro-5-oxohexanoate (4d). The batch reaction was carried out on a $0.2 \mathrm{mmol}$ scale, and the flow experiment was carried out on a $0.5 \mathrm{mmol}$ scale $(2.5 \mathrm{~mL}$ liquid was taken); $53.0 \mathrm{mg}$ were obtained in batch with a yield of $87 \%$, and 74.6 $\mathrm{mg}$ were obtained in flow with a yield of $93 \%$. The product was a pale yellow oil. Purification: Column chromatography $\left(\mathrm{CH}_{2} \mathrm{Cl}_{2} /\right.$ Acetone $=$ 20:1). ${ }^{1} \mathrm{H}$ NMR $\left(400 \mathrm{MHz}, \mathrm{CDCl}_{3}\right): \delta 8.31-8.30(\mathrm{~m}, 1 \mathrm{H}), 7.17(\mathrm{~d}, J$ $=1.5 \mathrm{~Hz}, 1 \mathrm{H}), 7.04(\mathrm{dd}, J=5.1,1.6 \mathrm{~Hz}, 1 \mathrm{H}), 4.17(\mathrm{dq}, J=7.2,4.2$ $\mathrm{Hz}, 2 \mathrm{H}$ ), 3.98 (dd, $J=7.3,5.7 \mathrm{~Hz}, 1 \mathrm{H}), 3.01$ (dtd, $J=18.5,15.2,7.4$ $\mathrm{Hz}, 1 \mathrm{H}), 2.26(\mathrm{dtd}, J=18.1,14.9,5.6 \mathrm{~Hz}, 1 \mathrm{H}), 2.08(\mathrm{~s}, 3 \mathrm{H}), 1.26(\mathrm{t}, J$ $=8.0 \mathrm{~Hz}, 3 \mathrm{H}) .{ }^{13} \mathrm{C}$ NMR $\left(100 \mathrm{MHz}, \mathrm{CDCl}_{3}\right): \delta 203.2,163.4(\mathrm{t}, J=$ $32.0 \mathrm{~Hz}), 152.4,150.4,149.2,123.8,121.9,114.7(\mathrm{t}, J=250.0 \mathrm{~Hz})$, 63.3, $51.3(\mathrm{t}, J=3.0 \mathrm{~Hz}), 36.2(\mathrm{t}, J=23.0 \mathrm{~Hz}), 29.2,13.8 .{ }^{19} \mathrm{~F}$ NMR $\left(376 \mathrm{MHz}, \mathrm{CDCl}_{3}\right): \delta-104.70(\mathrm{dd}, J=47.0,4.3 \mathrm{~Hz})$. HRMS (ESI) $m / z:[\mathrm{M}+\mathrm{Na}]^{+}$calcd for $\mathrm{C}_{13} \mathrm{H}_{14} \mathrm{ClF}_{2} \mathrm{NO}_{3} \mathrm{Na}$, 328.0528; found, 328.0520 .

Ethyl 2,2-Difluoro-5-oxo-4-(pyridin-3-yl)hexanoate (4e). The batch experiment was carried out on a $0.2 \mathrm{mmol}$ scale; $24.4 \mathrm{mg}$ of product were isolated as a yellow oil (45\% yield). Purification: Column chromatography $\left(\mathrm{CH}_{2} \mathrm{Cl}_{2} /\right.$ Acetone $\left.=20: 1\right) .{ }^{1} \mathrm{H}$ NMR $(400$ $\left.\mathrm{MHz}, \mathrm{CDCl}_{3}\right): \delta 8.58(\mathrm{~d}, J=8.0 \mathrm{~Hz}, 2 \mathrm{H}), 7.57(\mathrm{td}, J=8.0,1.9 \mathrm{~Hz}$, $1 \mathrm{H}), 7.35-7.32(\mathrm{~m}, 1 \mathrm{H}), 4.21(\mathrm{dq}, J=7.2,5.4 \mathrm{~Hz}, 2 \mathrm{H}), 4.09(\mathrm{t}, J=$ $4.0 \mathrm{~Hz}, 1 \mathrm{H}), 3.11(\mathrm{dtd}, J=16.7,15.1,7.3 \mathrm{~Hz}, 1 \mathrm{H}), 2.37(\mathrm{dtd}, J=$ $16.4,15.0,5.9 \mathrm{~Hz}, 1 \mathrm{H}), 2.14(\mathrm{~s}, 3 \mathrm{H}), 1.32(\mathrm{t}, J=8.0 \mathrm{~Hz}, 3 \mathrm{H}) .{ }^{13} \mathrm{C}$ NMR $\left(100 \mathrm{MHz}, \mathrm{CDCl}_{3}\right): \delta 204.5,163.6(\mathrm{t}, J=32.0 \mathrm{~Hz}), 149.4$, $149.0,135.7,133.3,124.2,114.9(\mathrm{t}, J=250.0 \mathrm{~Hz}), 63.2,49.7(\mathrm{t}, J=$ $4.0 \mathrm{~Hz}), 36.5(\mathrm{t}, J=24.0 \mathrm{~Hz}), 29.0,13.8 .{ }^{19} \mathrm{~F}$ NMR $(376 \mathrm{MHz}$, $\left.\mathrm{CDCl}_{3}\right): \delta-104.67(\mathrm{~s})$. HRMS (ESI) $\mathrm{m} / z:[\mathrm{M}+\mathrm{Na}]^{+}$calcd for $\mathrm{C}_{13} \mathrm{H}_{15} \mathrm{~F}_{2} \mathrm{NO}_{3} \mathrm{Na}$, 294.0918; found, 294.0918.

Ethyl 4-(6-Bromopyridin-3-yl)-2,2-difluoro-5-oxohexanoate (4f). The batch reaction was carried out on a $0.2 \mathrm{mmol}$ scale; $42.6 \mathrm{mg}$ of product were obtained as a pale yellow oil (61\% yield). Purification: Column chromatography $\left(\mathrm{CH}_{2} \mathrm{Cl}_{2} /\right.$ Acetone $\left.=30: 1\right) .{ }^{1} \mathrm{H}$ NMR $(400$ $\left.\mathrm{MHz}, \mathrm{CDCl}_{3}\right): \delta 8.31(\mathrm{~s}, 1 \mathrm{H}), 7.50(\mathrm{~d}, J=8.0 \mathrm{~Hz}, 1 \mathrm{H}), 7.40(\mathrm{~d}, J=$ $8.0,1 \mathrm{H}), 4.24(\mathrm{dq}, J=8.0,4.0 \mathrm{~Hz}, 2 \mathrm{H}), 4.06(\mathrm{t}, J=8.0 \mathrm{~Hz}, 1 \mathrm{H}), 3.08$ $(\mathrm{dtd}, J=17.6,15.5,7.3 \mathrm{~Hz}, 1 \mathrm{H}), 2.34(\mathrm{dtd}, J=17.2,15.3,5.9 \mathrm{~Hz}$, $1 \mathrm{H}), 2.15(\mathrm{~s}, 3 \mathrm{H}), 1.33(\mathrm{t}, J=8.0 \mathrm{~Hz}, 3 \mathrm{H}) .{ }^{13} \mathrm{C}$ NMR $(100 \mathrm{MHz}$, $\left.\mathrm{CDCl}_{3}\right): \delta 204.1,163.4(\mathrm{t}, J=32.0 \mathrm{~Hz}), 150.0,141.9,137.7,132.4$, $128.7,114.8(\mathrm{t}, J=250.0 \mathrm{~Hz}), 63.3,48.9(\mathrm{t}, J=4.0 \mathrm{~Hz}), 36.5(\mathrm{t}, J=$ $24.0 \mathrm{~Hz}), 29.1,13.8 .{ }^{19} \mathrm{~F}$ NMR $\left(376 \mathrm{MHz}, \mathrm{CDCl}_{3}\right): \delta-104.68(\mathrm{~s})$. HRMS (ESI) $m / z:[\mathrm{M}+\mathrm{Na}]^{+}$calcd for $\mathrm{C}_{13} \mathrm{H}_{14} \mathrm{BrF}_{2} \mathrm{NO}_{3} \mathrm{Na}$, 372.0023; found, 372.0026 .

Ethyl 4-(5-Bromopyridin-3-yl)-2,2-difluoro-5-oxohexanoate (4g). The batch reaction was carried out on a $0.2 \mathrm{mmol}$ scale, and the flow experiment was carried out on a $0.5 \mathrm{mmol}$ scale $(2.7 \mathrm{~mL}$ liquid was taken); $41.1 \mathrm{mg}$ and $62.2 \mathrm{mg}$ of product were obtained in batch and flow as a pale yellow oil (59\%, 66\% yields). Purification: Column chromatography $\left(\mathrm{CH}_{2} \mathrm{Cl}_{2} /\right.$ Acetone $\left.=30: 1\right) .{ }^{1} \mathrm{H} \mathrm{NMR}(400 \mathrm{MHz}$, $\left.\mathrm{CDCl}_{3}\right): \delta 8.63(\mathrm{~s}, 1 \mathrm{H}), 8.45(\mathrm{~s}, 1 \mathrm{H}), 7.69(\mathrm{~s}, 1 \mathrm{H}), 4.16(\mathrm{dq}, J=8.0$, $4.0 \mathrm{~Hz}, 2 \mathrm{H}), 4.00(\mathrm{t}, J=4.0 \mathrm{~Hz}, 1 \mathrm{H}), 3.05-2.97(\mathrm{~m}, 1 \mathrm{H}), 2.28(\mathrm{dtd}, J$ $=17.2,15.4,5.8 \mathrm{~Hz}, 1 \mathrm{H}), 2.09(\mathrm{~s}, 3 \mathrm{H}), 1.25(\mathrm{t}, J=8.0 \mathrm{~Hz}, 3 \mathrm{H}) .{ }^{13} \mathrm{C}$ $\operatorname{NMR}\left(100 \mathrm{MHz}, \mathrm{CDCl}_{3}\right): \delta 203.9,163.4(\mathrm{t}, J=32.0 \mathrm{~Hz}), 150.5$, $147.7,137.8,134.7,121.1,114.7(\mathrm{t}, J=250.0 \mathrm{~Hz}), 63.2,49.1(\mathrm{t}, J=$ $4.0 \mathrm{~Hz}), 36.5(\mathrm{t}, J=24.0 \mathrm{~Hz}), 29.1,13.8 .{ }^{19} \mathrm{~F}$ NMR $(376 \mathrm{MHz}$, $\mathrm{CDCl}_{3}$ ): $\delta-104.70(\mathrm{~s})$. HRMS (ESI) $\mathrm{m} / z:[\mathrm{M}+\mathrm{Na}]^{+}$calcd for $\mathrm{C}_{13} \mathrm{H}_{14} \mathrm{BrF}_{2} \mathrm{NO}_{3} \mathrm{Na}$, 372.0023; found, 372.0026. 
Ethyl 2,2-Difluoro-5-oxo-4-(pyridin-3-yl)heptanoate (4h). The batch reaction was carried out on a $0.2 \mathrm{mmol}$ scale, and the flow experiment was carried out on a $0.5 \mathrm{mmol}$ scale $(3.0 \mathrm{~mL}$ liquid was taken); $32.5 \mathrm{mg}$ and $52.1 \mathrm{mg}$ of product were obtained in batch and flow as a pale yellow oil (57\%, $61 \%$ yields). Purification: Column chromatography $\left(\mathrm{CH}_{2} \mathrm{Cl}_{2} /\right.$ Acetone $\left.=20: 1\right) .{ }^{1} \mathrm{H}$ NMR $(400 \mathrm{MHz}$, $\left.\mathrm{CDCl}_{3}\right): \delta 8.58(\mathrm{~d}, J=4.0 \mathrm{~Hz}, 2 \mathrm{H}), 7.56(\mathrm{~m}, 1 \mathrm{H}), 7.35-7.32(\mathrm{~m}$, $1 \mathrm{H}), 4.20(\mathrm{dq}, J=7.2,4.6 \mathrm{~Hz}, 2 \mathrm{H}), 4.08(\mathrm{dd}, J=7.6,5.6 \mathrm{~Hz}, 1 \mathrm{H})$, 3.19-3.05 (m, 1H), 2.54-2.37 (m, 1H), 2.36-2.30 (m, 1H), $1.32(\mathrm{t}$, $J=7.2 \mathrm{~Hz}, 3 \mathrm{H}), 0.99(\mathrm{t}, J=7.2 \mathrm{~Hz}, 3 \mathrm{H}) .{ }^{13} \mathrm{C}$ NMR $(100 \mathrm{MHz}$, $\left.\mathrm{CDCl}_{3}\right): \delta 207.5,163.6(\mathrm{t}, J=32.0 \mathrm{~Hz}), 149.5,149.1,135.4,133.5$, 124.0, $114.9(\mathrm{t}, J=250.0 \mathrm{~Hz}), 63.1,49.6,36.8(\mathrm{t}, J=24.0 \mathrm{~Hz}), 35.1$, 13.8. $\left.{ }^{19} \mathrm{~F} \mathrm{NMR} \mathrm{(376} \mathrm{MHz,} \mathrm{CDCl}_{3}\right): \delta-104.76$ (s). HRMS (ESI) $\mathrm{m} /$ $z:[\mathrm{M}+\mathrm{Na}]^{+}$calcd for $\mathrm{C}_{14} \mathrm{H}_{17} \mathrm{~F}_{2} \mathrm{NO}_{3} \mathrm{Na}, 308.1074$; found, 308.1079.

Ethyl 2,2-Difluoro-5-oxo-4-(pyridin-2-yl)decanoate (4i). The batch reaction was carried out on a $0.2 \mathrm{mmol}$ scale; $40.5 \mathrm{mg}$ of product were obtained as a pale yellow oil (62\% yield). Purification: Column chromatography $\left(\mathrm{CH}_{2} \mathrm{Cl}_{2} /\right.$ Acetone $\left.=16: 1\right)$. ${ }^{1} \mathrm{H}$ NMR $(400$ $\left.\mathrm{MHz}, \mathrm{CDCl}_{3}\right): \delta 8.56(\mathrm{~d}, J=4.0 \mathrm{~Hz}, 1 \mathrm{H}), 7.67(\mathrm{td}, J=8.0,4.0 \mathrm{~Hz}$, $1 \mathrm{H}), 7.25(\mathrm{~s}, 1 \mathrm{H}), 7.20(\mathrm{ddd}, J=8.0,4.0,1.1 \mathrm{~Hz}, 1 \mathrm{H}), 4.26-4.16(\mathrm{~m}$, $3 \mathrm{H}), 3.21-3.03(\mathrm{~m}, 1 \mathrm{H}), 2.57(\mathrm{dtd}, J=18.4,15.3,6.1 \mathrm{~Hz}, 1 \mathrm{H})$, $2.47-2.24(\mathrm{~m}, 2 \mathrm{H}), 1.48(\mathrm{dtd}, J=14.9,8.2,6.7 \mathrm{~Hz}, 2 \mathrm{H}), 1.30(\mathrm{t}, J=$ $8.0 \mathrm{~Hz}, 3 \mathrm{H}), 1.24-1.08(\mathrm{~m}, 4 \mathrm{H}), 0.80(\mathrm{t}, J=7.1 \mathrm{~Hz}, 3 \mathrm{H}) .{ }^{13} \mathrm{C}$ NMR $\left(100 \mathrm{MHz}, \mathrm{CDCl}_{3}\right): \delta 206.5,163.8(\mathrm{t}, J=33.0 \mathrm{~Hz}), 157.2,149.6$, $137.4,123.5,122.6,115.4(\mathrm{t}, J=249.0 \mathrm{~Hz}), 63.0,53.9,41.5,35.6(\mathrm{t}, J$ $=23.0 \mathrm{~Hz}), 23.2,22.3,13.8 .{ }^{19} \mathrm{~F} \mathrm{NMR}\left(376 \mathrm{MHz}, \mathrm{CDCl}_{3}\right): \delta-104.72$ (s). HRMS (ESI) $m / z:[\mathrm{M}+\mathrm{Na}]^{+}$calcd for $\mathrm{C}_{17} \mathrm{H}_{23} \mathrm{~F}_{2} \mathrm{NO}_{3} \mathrm{Na}$, 350.1544; found, 350.1548 .

Ethyl 2,2-Difluoro-5-oxo-4-(pyrazin-2-yl)hexanoate (4j). The batch reaction was carried out on a $0.2 \mathrm{mmol}$ scale, and the flow experiment was carried out on a $0.5 \mathrm{mmol}$ scale $(2.8 \mathrm{~mL}$ liquid was taken); $42.5 \mathrm{mg}$ and $69.3 \mathrm{mg}$ of product were obtained in batch and flow as a pale yellow oil $(78 \%, 91 \%$ yields). Purification: Column chromatography $\mathrm{CH}_{2} \mathrm{Cl}_{2} /$ Acetone $\left.=20: 1\right) .{ }^{1} \mathrm{H}$ NMR $(400 \mathrm{MHz}$, $\left.\mathrm{CDCl}_{3}\right): \delta 8.63(\mathrm{~s}, 1 \mathrm{H}), 8.57-8.54(\mathrm{~m}, 2 \mathrm{H}), 4.30-4.23(\mathrm{~m}, 3 \mathrm{H})$, $3.22-3.08(\mathrm{~m}, 1 \mathrm{H}), 2.71-2.57(\mathrm{~m}, 1 \mathrm{H}), 2.13(\mathrm{~s}, 3 \mathrm{H}), 1.33(\mathrm{t}, J=8.0$ $\mathrm{Hz}, 3 \mathrm{H}) .{ }^{13} \mathrm{C}$ NMR $\left(100 \mathrm{MHz}, \mathrm{CDCl}_{3}\right): \delta 202.9,163.6(\mathrm{t}, J=22.0$ $\mathrm{Hz}), 153.1,144.9,144.6,143.7,115.2(\mathrm{t}, J=250.0 \mathrm{~Hz}), 63.2,52.2$, $35.0(\mathrm{t}, J=23.0 \mathrm{~Hz}), 28.9,13.9 .{ }^{19} \mathrm{~F}$ NMR $\left(376 \mathrm{MHz}, \mathrm{CDCl}_{3}\right): \delta$ -104.33 to $-105.72(\mathrm{~m})$. HRMS (ESI) $\mathrm{m} / z$ : $[\mathrm{M}+\mathrm{Na}]^{+}$calcd for $\mathrm{C}_{12} \mathrm{H}_{14} \mathrm{~F}_{2} \mathrm{~N}_{2} \mathrm{O}_{3} \mathrm{Na}$, 295.0870; found, 295.0875.

Ethyl 2,2-Difluoro-4-(3-methylpyrazin-2-yl)-5-oxohexanoate (4k). The batch experiment was carried out on a $0.2 \mathrm{mmol}$ scale; $40.5 \mathrm{mg}$ of product were isolated as a yellow oil ( $71 \%$ yield). Purification: Column chromatography $\left(\mathrm{CH}_{2} \mathrm{Cl}_{2}\right.$ /Acetone $\left.=16: 1\right) .{ }^{1} \mathrm{H}$ NMR $\left(400 \mathrm{MHz}, \mathrm{CDCl}_{3}\right): 8.62-8.27(\mathrm{~m}, 2 \mathrm{H}), 4.37(\mathrm{t}, J=8.0 \mathrm{~Hz}$, $1 \mathrm{H}), 4.23(\mathrm{dq}, J=7.2,3.0 \mathrm{~Hz}, 2 \mathrm{H}), 3.11(\mathrm{dtd}, J=20.7,15.5,5.3 \mathrm{~Hz}$, $1 \mathrm{H}), 2.72(\mathrm{~s}, 3 \mathrm{H}), 2.69-2.54(\mathrm{~m}, 1 \mathrm{H}), 2.03(\mathrm{~s}, 3 \mathrm{H}), 1.32(\mathrm{t}, J=7.2$ $\mathrm{Hz}, 3 \mathrm{H}) .{ }^{13} \mathrm{C}$ NMR $\left(100 \mathrm{MHz}, \mathrm{CDCl}_{3}\right): \delta 202.5,163.6(\mathrm{t}, J=32.0$ $\mathrm{Hz}), 152.9,151.8,142.7,142.1,115.3(\mathrm{t}, J=250.0 \mathrm{~Hz}), 63.1,50.5(\mathrm{t}, J$ $=2.0 \mathrm{~Hz}), 34.8(\mathrm{t}, J=23.0 \mathrm{~Hz}), 29.7,28.4,21.7,13.8 .{ }^{19} \mathrm{~F}$ NMR $(376$ $\mathrm{MHz}, \mathrm{CDCl}_{3}$ ): $\delta-104.97$ (ddd, $J=64.6,20.0,14.9 \mathrm{~Hz}$ ). HRMS (ESI) $\mathrm{m} / z:[\mathrm{M}+\mathrm{Na}]^{+}$calcd for $\mathrm{C}_{13} \mathrm{H}_{16} \mathrm{~F}_{2} \mathrm{~N}_{2} \mathrm{O}_{3} \mathrm{Na}$, 309.1027; found, 309.1034.

Ethyl 4-(Benzo[b]thiophen-2-yl)-2,2-difluoro-5-oxohexanoate (4I). The flow experiment was carried out on a $0.5 \mathrm{mmol} \mathrm{scale}$, and $2.2 \mathrm{~mL}$ of liquid were collected; $60.2 \mathrm{mg}$ of product were isolated as a pale yellow oil ( $84 \%$ yield). Purification: Column chromatography (Cyclohexane/EtOAc $=50: 1$ to $40: 1) .{ }^{1} \mathrm{H} \mathrm{NMR}\left(400 \mathrm{MHz}, \mathrm{CDCl}_{3}\right)$ : $\delta 7.81-7.71(\mathrm{~m}, 2 \mathrm{H}), 7.38-7.31(\mathrm{~m}, 2 \mathrm{H}), 7.17(\mathrm{~s}, 1 \mathrm{H}), 4.37(\mathrm{dd}, J=$ 7.7, $5.3 \mathrm{~Hz}, 1 \mathrm{H}$ ), 4.18 (qq, $J=10.8,7.2 \mathrm{~Hz}, 2 \mathrm{H}), 3.19$ (dtd, $J=17.2$, $15.4,7.8 \mathrm{~Hz}, 1 \mathrm{H}), 2.55-2.49(\mathrm{~m}, 1 \mathrm{H}), 2.24(\mathrm{~s}, 3 \mathrm{H}), 1.29(\mathrm{t}, J=8.0$ $\mathrm{Hz}, 3 \mathrm{H}) .{ }^{13} \mathrm{C}$ NMR $\left(100 \mathrm{MHz}, \mathrm{CDCl}_{3}\right): \delta 203.6,163.6(\mathrm{t}, J=32.0$ $\mathrm{Hz}), 139.7(\mathrm{t}, J=23.0 \mathrm{~Hz}), 124.7,123.5,122.3,114.9(\mathrm{t}, J=250.0$ $\mathrm{Hz}), 63.1,47.8,37.0(\mathrm{t}, J=24.0 \mathrm{~Hz}), 28.6,13.8 .{ }^{19} \mathrm{~F} \mathrm{NMR}(376 \mathrm{MHz}$, $\left.\mathrm{CDCl}_{3}\right): \delta-104.77(\mathrm{~d}, J=40.4 \mathrm{~Hz})$. HRMS $(\mathrm{ESI}) \mathrm{m} / z:[\mathrm{M}+\mathrm{Na}]^{+}$ calcd for $\mathrm{C}_{16} \mathrm{H}_{16} \mathrm{~F}_{2} \mathrm{O}_{3} \mathrm{SNa}$, 349.0686; found, 349.0674 .

Ethyl 4-(7-(2-Ethoxy-1,1-difluoro-2-oxoethyl)benzofuran-2-yl)2,2-difluoro-5-oxohexanoate $(4 \mathrm{~m})$. The batch reaction was carried out on a $0.2 \mathrm{mmol}$ scale, and the flow experiment was carried out on a
$0.5 \mathrm{mmol}$ scale $(2.2 \mathrm{~mL}$ liquid was taken); $18.2 \mathrm{mg}$ and $33.0 \mathrm{mg}$ of product were obtained in batch and flow as a pale yellow oil $(21 \%$, $34 \%$ yields). Purification: Column chromatography with (Cyclohexane/EtOAc $=30: 1) .{ }^{1} \mathrm{H}$ NMR $\left(400 \mathrm{MHz}, \mathrm{CDCl}_{3}\right): \delta 7.58(\mathrm{~d}, J=$ $4.0 \mathrm{~Hz}, 1 \mathrm{H}), 7.49(\mathrm{~d}, J=8.0 \mathrm{~Hz}, 1 \mathrm{H}), 7.39-7.35(\mathrm{~m}, 1 \mathrm{H}), 6.92(\mathrm{~s}$, $1 \mathrm{H}), 4.33-4.27(\mathrm{~m}, 3 \mathrm{H}), 4.26-4.14(\mathrm{~m}, 2 \mathrm{H}), 3.26-3.07(\mathrm{~m}, 1 \mathrm{H})$, $2.67-2.55(\mathrm{~m}, 1 \mathrm{H}), 2.24(\mathrm{~s}, 3 \mathrm{H}), 1.31-1.29(\mathrm{~m}, 6 \mathrm{H}) .{ }^{13} \mathrm{C}$ NMR $(100$ $\left.\mathrm{MHz}_{\mathrm{CDCl}}\right): \delta 201.8,163.5,155.2,154.4,125.5,124.4,120.7(\mathrm{t}, J=$ $7.0 \mathrm{~Hz}), 114.0,104.9,62.3,62.2,46.4,34.0,33.8,28.6,22.3,13.9$, 13.8. ${ }^{19} \mathrm{~F}$ NMR $\left(376 \mathrm{MHz}, \mathrm{CDCl}_{3}\right): \delta-102.95(\mathrm{~d}, J=41.1 \mathrm{~Hz})$, -105.06 (s). HRMS (ESI) $m / z:[\mathrm{M}+\mathrm{Na}]^{+}$calcd for $\mathrm{C}_{20} \mathrm{H}_{20} \mathrm{~F}_{4} \mathrm{O}_{6} \mathrm{Na}$, 455.1094; found, 455.1094.

Ethyl 4-(4-(2-Ethoxy-1,1-difluoro-2-oxoethyl)-7-methoxybenzofuran-2-yl)-2,2-difluoro-5-oxohexanoate (4n). The batch reaction was carried out on a $0.2 \mathrm{mmol}$ scale; $59.0 \mathrm{mg}$ of product were obtained in batch and flow as a pale yellow oil (64\% yield). Purification: Column chromatography (Cyclohexane $/$ EtOAc $=30: 1)$. ${ }^{1} \mathrm{H}$ NMR $\left(400 \mathrm{MHz}, \mathrm{CDCl}_{3}\right): \delta 7.42(\mathrm{~d}, J=8.0 \mathrm{~Hz}, 1 \mathrm{H}), 6.88(\mathrm{~s}$, $1 \mathrm{H}), 6.83(\mathrm{~d}, J=8.4 \mathrm{~Hz}, 1 \mathrm{H}), 4.32-4.26(\mathrm{~m}, 3 \mathrm{H}), 4.25-4.16(\mathrm{~m}$, $2 \mathrm{H}), 4.03(\mathrm{~s}, 3 \mathrm{H}), 3.22-3.08(\mathrm{~m}, 1 \mathrm{H}), 2.69-2.55(\mathrm{~m}, 1 \mathrm{H}), 2.24(\mathrm{~s}$, $3 \mathrm{H}), 1.32-1.67(\mathrm{~m}, 6 \mathrm{H}) .{ }^{13} \mathrm{C} \mathrm{NMR}\left(100 \mathrm{MHz}, \mathrm{CDCl}_{3}\right): \delta 201.7$, $164.0(\mathrm{t}, J=36.0 \mathrm{~Hz}), 163.5(\mathrm{t}, J=32.0 \mathrm{~Hz}), 154.5,147.2,144.3$, $127.2,122.1,117.3(\mathrm{t}, J=27.0 \mathrm{~Hz}), 116.2,114.9(\mathrm{t}, J=250.0 \mathrm{~Hz})$, $113.7(\mathrm{t}, J=251.0 \mathrm{~Hz}), 106.1,105.2,70.6,63.1,56.2,46.3(\mathrm{t}, J=3.0$ $\mathrm{Hz}), 34.0(\mathrm{t}, J=24.0 \mathrm{~Hz}), 28.6,26.5,23.2,22.3,13.9,13.8$. ${ }^{19} \mathrm{~F}$ NMR $\left(376 \mathrm{MHz}, \mathrm{CDCl}_{3}\right): \delta-102.07(\mathrm{~d}, J=41.1 \mathrm{~Hz}),-105.06(\mathrm{~s})$. HRMS (ESI) $m / z:[\mathrm{M}+\mathrm{Na}]^{+}$calcd for $\mathrm{C}_{21} \mathrm{H}_{22} \mathrm{~F}_{4} \mathrm{O}_{7} \mathrm{Na}$, 485.1199; found, 485.1194 .

Ethyl 4-(3-(2-Ethoxy-1,1-difluoro-2-oxoethyl)thiophen-2-yl)-2,2difluoro-5-oxohexanoate (40). The batch experiment was carried out on a $0.2 \mathrm{mmol}$ scale; $56.5 \mathrm{mg}$ product were isolated as a yellow oil (71\% yield). Purification: Column chromatography (Cyclohexane/ EtOAc $=20: 1$ to $8: 1) .{ }^{1} \mathrm{H}$ NMR $\left(400 \mathrm{MHz}, \mathrm{CDCl}_{3}\right): \delta 7.26-7.25(\mathrm{~m}$, $1 \mathrm{H}), 6.89-6.87(\mathrm{~m}, 1 \mathrm{H}), 4.37(\mathrm{q}, J=7.1 \mathrm{~Hz}, 2 \mathrm{H}), 4.33-4.30(\mathrm{~m}$, $1 \mathrm{H}), 4.27-4.19(\mathrm{~m}, 2 \mathrm{H}), 3.11(\mathrm{dtd}, J=17.5,15.2,8.0 \mathrm{~Hz}, 1 \mathrm{H})$, $2.51-2.29(\mathrm{~m}, 1 \mathrm{H}), 2.21(\mathrm{~s}, 3 \mathrm{H}), 1.38-1.31(\mathrm{~m}, 6 \mathrm{H}) .{ }^{13} \mathrm{C} \mathrm{NMR}(100$ $\left.\mathrm{MHz}, \mathrm{CDCl}_{3}\right): \delta 203.3,163.4(\mathrm{t}, J=32.0 \mathrm{~Hz}), 163.0(\mathrm{t}, J=35.0 \mathrm{~Hz})$, $143.3(\mathrm{t}, J=2.0 \mathrm{~Hz}), 134.1(\mathrm{t}, J=30.0 \mathrm{~Hz}), 128.7(\mathrm{t}, J=5.0 \mathrm{~Hz})$, $126.4,114.6(\mathrm{t}, J=250.0 \mathrm{~Hz}), 111.2(\mathrm{t}, J=250.0 \mathrm{~Hz}), 63.6,63.2$, $47.0,37.5(\mathrm{t}, J=24.0 \mathrm{~Hz}), 28.6,13.9,13.8 .{ }^{19} \mathrm{~F}$ NMR $(376 \mathrm{MHz}$, $\left.\mathrm{CDCl}_{3}\right): \delta-93.05$ to $-93.13(\mathrm{~m}),-104.81$ to $-105.0(\mathrm{~m})$. HRMS (ESI) $m / z:[\mathrm{M}+\mathrm{Na}]^{+}$calcd for $\mathrm{C}_{16} \mathrm{H}_{18} \mathrm{~F}_{4} \mathrm{O}_{5} \mathrm{SNa}$, 421.0709; found, 421.0713

Diethyl (3-(Benzo[b]thiophen-2-yl)-1,1-difluoro-4-oxopentyl)phosphonate (5a). The flow experiment was carried out on a 0.5 mmol scale, and $2.5 \mathrm{~mL}$ of liquid were collected; $81.2 \mathrm{mg}$ of product were isolated as a colorless oil (84\% yield). Purification: Column chromatography (Cyclohexane/EtOAc $=8: 1$ to $2: 1) .{ }^{1} \mathrm{H}$ NMR $(400$ $\left.\mathrm{MHz}, \mathrm{CDCl}_{3}\right): \delta 7.76(\mathrm{dd}, J=20.0,8.0 \mathrm{~Hz}, 2 \mathrm{H}), 7.37-7.30(\mathrm{~m}, 2 \mathrm{H})$, $7.18(\mathrm{~s}, 1 \mathrm{H}), 4.54(\mathrm{q}, J=4.0 \mathrm{~Hz}, 1 \mathrm{H}), 4.32-4.24(\mathrm{~m}, 4 \mathrm{H}), 3.37-3.20$ (m, $1 \mathrm{H}), 2.53-2.40(\mathrm{~m}, 1 \mathrm{H}), 2.27(\mathrm{~s}, 3 \mathrm{H}), 1.39(\mathrm{q}, J=8.0 \mathrm{~Hz}, 6 \mathrm{H})$. ${ }^{13} \mathrm{C}$ NMR $\left(100 \mathrm{MHz}, \mathrm{CDCl}_{3}\right): \delta 203.8,140.8,139.6,139.5,124.6$, $124.5,123.4,122.9,122.3,120.7(\mathrm{t}, J=259.0 \mathrm{~Hz}), 118.6(\mathrm{t}, J=260.0$ $\mathrm{Hz}), 64.7(\mathrm{t}, J=6.0 \mathrm{~Hz}), 47.0(\mathrm{q}, J=5.0 \mathrm{~Hz}), 36.6(\mathrm{td}, J=20.0,5.0$ $\mathrm{Hz}), 28.7,16.4,16.3 .{ }^{19} \mathrm{~F} \mathrm{NMR}\left(376 \mathrm{MHz}, \mathrm{CDCl}_{3}\right): \delta-111.81-$ $109.54(\mathrm{~m})$. HRMS (ESI) $\mathrm{m} / z$ : $[\mathrm{M}+\mathrm{H}]^{+}$calcd for $\mathrm{C}_{17} \mathrm{H}_{21} \mathrm{~F}_{2} \mathrm{O}_{4} \mathrm{PS}$, 391.0944; found, 391.0945.

4-(Benzo[b]thiophen-2-yl)-2,2-difluoro-1-morpholinohexane1,5 -dione $(5 b)$. The flow experiment was carried out on a $0.5 \mathrm{mmol}$ scale, and $2.6 \mathrm{~mL}$ of liquid were collected; $83.0 \mathrm{mg}$ of product were isolated as a colorless oil ( $88 \%$ yield). Purification: Column chromatography (Cyclohexane/EtOAc $=30: 1$ to $16: 1) .{ }^{1} \mathrm{H}$ NMR $\left(400 \mathrm{MHz}, \mathrm{CDCl}_{3}\right): \delta 7.81-7.71(\mathrm{~m}, 2 \mathrm{H}), 7.38-7.30(\mathrm{~m}, 2 \mathrm{H}), 7.17$ $(\mathrm{s}, 1 \mathrm{H}), 4.45(\mathrm{q}, J=4.0 \mathrm{~Hz}, 1 \mathrm{H}), 3.74-3.61(\mathrm{~m}, 8 \mathrm{H}), 3.31$ (dddd, $J=$ $18.3,16.8,15.1,8.1 \mathrm{~Hz}, 1 \mathrm{H}), 2.64-2.50(\mathrm{~m}, 1 \mathrm{H}), 2.25(\mathrm{~s}, 3 \mathrm{H}) .{ }^{13} \mathrm{C}$ NMR $\left(100 \mathrm{MHz}, \mathrm{CDCl}_{3}\right): \delta 204.0,161.5(\mathrm{t}, J=28.0 \mathrm{~Hz}), 140.8$, $139.6,139.5,124.6,124.5,123.5,122.9,122.3,118.2(\mathrm{t}, J=253.0$ $\mathrm{Hz}), 100.0,66.6,48.0,46.5,43.3,37.4(\mathrm{t}, J=22.0 \mathrm{~Hz}), 28.7,26.9 .{ }^{19} \mathrm{~F}$ NMR $\left(376 \mathrm{MHz}, \mathrm{CDCl}_{3}\right): \delta-98.02(\mathrm{~s})$. HRMS (ESI) $\mathrm{m} / z$ : $[\mathrm{M}+$ $\mathrm{Na}]^{+}$calcd for $\mathrm{C}_{18} \mathrm{H}_{19} \mathrm{~F}_{2} \mathrm{NO}_{3} \mathrm{SNa}, 390.0951$; found, 390.0945 . 
4-(Benzo[b]thiophen-2-yl)-2,2-difluoro-1-(piperidin-1-yl)hexane1,5-dione (5c). The flow experiment was carried out on a $0.5 \mathrm{mmol}$ scale, and $2.8 \mathrm{~mL}$ of liquid were collected; $89.4 \mathrm{mg}$ of product were isolated as a colorless oil (94\% yield). Purification: Column chromatography (Cyclohexane/EtOAc $=30: 1$ to $16: 1$ ). ${ }^{1} \mathrm{H}$ NMR $\left(400 \mathrm{MHz}, \mathrm{CDCl}_{3}\right): \delta 7.79-7.70(\mathrm{~m}, 2 \mathrm{H}), 7.36-7.27(\mathrm{~m}, 2 \mathrm{H}), 7.17$ $(\mathrm{s}, 1 \mathrm{H}), 4.46(\mathrm{dd}, J=8.0,4.0 \mathrm{~Hz}, 1 \mathrm{H}), 3.57(\mathrm{td}, J=20.0,4.0 \mathrm{~Hz}, 4 \mathrm{H})$, 3.31 (dddd, $J=18.3,16.8,15.1,8.1 \mathrm{~Hz}, 1 \mathrm{H}$ ), 2.56 (dddd, $J=18.3$, $16.8,15.1,8.1 \mathrm{~Hz}, 1 \mathrm{H}), 2.24(\mathrm{~s}, 3 \mathrm{H}), 1.65-1.56(\mathrm{~m}, 6 \mathrm{H}) .{ }^{13} \mathrm{C} \mathrm{NMR}$ $\left(100 \mathrm{MHz}, \mathrm{CDCl}_{3}\right): \delta 204.2,161.1(\mathrm{t}, J=28.0 \mathrm{~Hz}), 141.0,139.6$, $139.5,124.5,124.3,123.4,122.7,122.2,118.4(\mathrm{t}, J=253.0 \mathrm{~Hz}), 48.1$ $(\mathrm{t}, J=4.0 \mathrm{~Hz}), 46.7(\mathrm{t}, J=6.0 \mathrm{~Hz}), 44.3,37.6(\mathrm{t}, J=23.0 \mathrm{~Hz}), 28.6$, 26.3, 25.5, 24.3. ${ }^{19} \mathrm{~F}$ NMR (376 MHz, $\left.\mathrm{CDCl}_{3}\right): \delta-98.0(\mathrm{~s})$. HRMS (ESI) $\mathrm{m} / z:[\mathrm{M}+\mathrm{Na}]^{+}$calcd for $\mathrm{C}_{19} \mathrm{H}_{21} \mathrm{~F}_{2} \mathrm{NO}_{2} \mathrm{SNa}, 388.1159$; found, 388.1155 .

4-(Benzo[b]thiophen-2-yl)-2,2-difluoro-1-(pyrrolidin-1-yl)hexane-1,5-dione $(5 d)$. The flow experiment was carried out on a 0.5 mmol scale, and $2.2 \mathrm{~mL}$ of liquid were collected; $65.4 \mathrm{mg}$ of product were isolated as a colorless oil ( $85 \%$ yield). Purification: Column chromatography (Cyclohexane/EtOAc $=30: 1$ to $16: 1) .{ }^{1} \mathrm{H}$ NMR $\left(400 \mathrm{MHz}, \mathrm{CDCl}_{3}\right): \delta 7.79-7.70(\mathrm{dd}, J=28.0,8.0 \mathrm{~Hz}, 2 \mathrm{H}), 7.36-$ $7.29(\mathrm{~m}, 2 \mathrm{H}), 7.16(\mathrm{~s}, 1 \mathrm{H}), 4.48(\mathrm{q}, J=4.0 \mathrm{~Hz}, 1 \mathrm{H}), 3.64(\mathrm{q}, J=4.0$ $\mathrm{Hz}, 2 \mathrm{H}), 3.46(\mathrm{q}, J=6.8 \mathrm{~Hz}, 2 \mathrm{H}), 3.26(\mathrm{tdd}, J=17.4,14.9,8.3 \mathrm{~Hz}$, $1 \mathrm{H}), 2.55(\mathrm{qd}, J=16.3,4.3 \mathrm{~Hz}, 1 \mathrm{H}), 2.24(\mathrm{~s}, 3 \mathrm{H}), 1.97-1.91(\mathrm{~m}$, $2 \mathrm{H}), 1.86-1.78(\mathrm{~m}, 2 \mathrm{H}) .{ }^{13} \mathrm{C}$ NMR $\left(100 \mathrm{MHz}, \mathrm{CDCl}_{3}\right): \delta 204.2$, $161.6(\mathrm{t}, J=29.0 \mathrm{~Hz}), 140.8,139.6,139.5,124.5,124.4,123.4,122.8$, $122.2,117.6(\mathrm{t}, J=252.0 \mathrm{~Hz}), 48.0(\mathrm{t}, J=4.0 \mathrm{~Hz}), 47.4,46.6(\mathrm{t}, J=$ $23.0 \mathrm{~Hz}), 37.0(\mathrm{t}, J=24.0 \mathrm{~Hz}), 28.6,26.9,26.4,23.2 .{ }^{19} \mathrm{~F}$ NMR $(376$ $\left.\mathrm{MHz}, \mathrm{CDCl}_{3}\right): \delta-101.50(\mathrm{~s})$. HRMS (ESI) $\mathrm{m} / z:[\mathrm{M}+\mathrm{Na}]^{+}$calcd for $\mathrm{C}_{18} \mathrm{H}_{19} \mathrm{~F}_{2} \mathrm{NO}_{2} \mathrm{SNa}$, 374.1002; found, 374.1003.

\section{ASSOCIATED CONTENT}

\section{S Supporting Information}

The Supporting Information is available free of charge on the ACS Publications website at DOI: 10.1021/acs.joc.8b01624.

Description of reaction setups, radical trapping experiments, and spectral data of all products (PDF)

\section{AUTHOR INFORMATION}

\section{Corresponding Author}

*E-mail: t.noel@tue.nl.

\section{ORCID}

Timothy Noël: 0000-0002-3107-6927

\section{Notes}

The authors declare no competing financial interest.

\section{ACKNOWLEDGMENTS}

X.J.W. and T.N. would like to acknowledge the European Union for a Marie Curie ITN Grant (Photo4Future, Grant No. 641861). We also acknowledge the Dutch Science Foundation (NWO) for a VIDI grant for T.N. (SensPhotoFlow, No. 14150).

\section{REFERENCES}

(1) (a) Murakami, K.; Yamada, S.; Kaneda, T.; Itami, K. C-H Functionalization of Azines. Chem. Rev. 2017, 117, 9302-9332. (b) Yang, Y.; Lan, J.; You, J. Oxidative $\mathrm{C}-\mathrm{H} / \mathrm{C}-\mathrm{H}$ Coupling Reactions between Two (Hetero)arenes. Chem. Rev. 2017, 117, 8787-8863. (c) Boubertakh, O.; Goddard, J.-P. Construction and Functionalization of Heteroarenes by Use of Photoredox Catalysis. Eur. J. Org. Chem. 2017, 2017, 2072-2084. (d) Rossi, R.; Bellina, F.; Lessi, M.; Manzini, C. Cross-Coupling of Heteroarenes by C-H Functionalization: Recent Progress towards Direct Arylation and Heteroarylation Reactions Involving Heteroarenes Containing One Heteroatom. Adv. Synth. Catal. 2014, 356, 17-117. (e) Vitaku, E.; Smith, D. T.; Njardarson, J. T. Analysis of the Structural Diversity,
Substitution Patterns, and Frequency of Nitrogen Heterocycles among U.S. FDA Approved Pharmaceuticals. J. Med. Chem. 2014, 57, 10257-10274.

(2) (a) Twigg, D. G.; Kondo, N.; Mitchell, S. L.; Galloway, W. R. J. D.; Sore, H. F.; Madin, A.; Spring, D. R. Partially Saturated Bicyclic Heteroaromatics as an $\mathrm{sp}^{3}$-Enriched Fragment Collection. Angew. Chem., Int. Ed. 2016, 55, 12479-12483. (b) Birudukota, N. V. S.; Franke, R.; Hofer, B. An Approach to "Escape from Flatland": Chemo-enzymatic Synthesis and Biological Profiling of a Library of Bridged Bicyclic Compounds. Org. Biomol. Chem. 2016, 14, 38213837. (c) Lovering, F. Escape from Flatland 2: Complexity and Promiscuity. MedChemComm 2013, 4, 515-519. (d) Lovering, F.; Bikker, J.; Humblet, C. Escape from Flatland: Increasing Saturation as an Approach to Improving Clinical Success. J. Med. Chem. 2009, 52, 6752-6756.

(3) Duncton, M. A. J. Minisci Reactions: Versatile CHFunctionalizations for Medicinal Chemists. MedChemComm 2011, $2,1135-1161$.

(4) Ackermann, L. Metal-catalyzed Direct Alkylations of (Hetero)arenes via $\mathrm{C}-\mathrm{H}$ Nond Cleavages with Unactivated Alkyl Halides. Chem. Commun. 2010, 46, 4866-4877.

(5) For selected reviews on radical aryl migration reactions, see: (a) Zeng, Y.; Ni, C.; Hu, J. Recent Advances in the One-Step Synthesis of Distally Fluorinated Ketones. Chem. - Eur. J. 2016, 22, 3210-3223. (b) Chen, Z.-M.; Zhang, X.-M.; Tu, Y.-Q. Radical Aryl Migration Reactions and Synthetic Applications. Chem. Soc. Rev. 2015, 44, 5220-5245. (c) Studer, A.; Bossart, M. Radical Aryl Migration Reactions. Tetrahedron 2001, 57, 9649-9667.

(6) For some selected recent examples on photocatalytic migration chemistry, see: (a) Wu, X.; Wang, M.; Huan, L.; Wang, D.; Wang, J.; Zhu, C. Tertiary-Alcohol-Directed Functionalization of Remote $\mathrm{C}\left(\mathrm{sp}^{3}\right)-\mathrm{H}$ Bonds by Sequential Hydrogen Atom and Heteroaryl Migrations. Angew. Chem., Int. Ed. 2018, 57, 1640-1644. (b) Tang, X.; Studer, A. Alkene 1,2-Difunctionalization by Radical Alkenyl Migration. Angew. Chem., Int. Ed. 2018, 57, 814-817. (c) Yu, J.; Wang, D.; Xu, Y.; Wu, Z.; Zhu, C. Distal Functional Group Migration for Visible-light Induced Carbo-difluoroalkylation/monofluoroalkylation of Unactivated Alkenes. Adv. Synth. Catal. 2018, 360, 744-750. (d) Xu, Y.; Wu, Z.; Jiang, J.; Ke, Z.; Zhu, C. Merging Distal Alkynyl Migration and Photoredox Catalysis for Radical Trifluoromethylative Alkynylation of Unactivated Olefins. Angew. Chem., Int. Ed. 2017, 56, 4545-4548.

(7) For the handling of exothermic reactions in flow, see: (a) Laudadio, G.; Gemoets, H. P. L.; Hessel, V.; Noël, T. Flow Synthesis of Diaryliodonium Triflates. J. Org. Chem. 2017, 82, 1173511741. (b) Kockmann, N.; Thenée, P.; Fleischer-Trebes, C.; Laudadio, G.; Noël, T. Safety Assessment in Development and Operation of Modular Continuous-Flow Processes. React. Chem. Eng. 2017, 2, 258-280. (c) Gutmann, B.; Kappe, C. O. Forbidden Chemistries - Paths to a Sustainable Future Engaging Continuous Processsing. J. Flow Chem. 2017, 7, 65-71. (d) Movsisyan, M.; Delbeke, E. I. P.; Berton, J. K. E. T.; Battilocchio, C.; Ley, S. V.; Stevens, C. V. Taming Hazardous Chemistry by Continuous Flow Technology. Chem. Soc. Rev. 2016, 45, 4892-4928. (e) Gutmann, B.; Cantillo, D.; Kappe, C. O. Continuous-Flow Technology-A Tool for the Safe Manufacturing of Active Pharmaceutical Ingredients. Angew. Chem., Int. Ed. 2015, 54, 6688-6728. (f) Newby, J. A.; Huck, L.; Blaylock, W.; Witt, P. M.; Ley, S. V.; Browne, D. L. Investigation of a Lithium-Halogen Exchange Flow Process for the Preparation of Boronates by Using a Cryo-Flow Reactor. Chem. - Eur. J. 2014, 20, 263-271. (g) Newby, J. A.; Blaylock, D. W.; Witt, P. M.; Pastre, J. C.; Zacharova, M. K.; Ley, S. V.; Browne, D. L. Design and Application of a Low-Temperature Continuous Flow Chemistry Platform. Org. Process Res. Dev. 2014, 18, 1211-1220. (h) Newby, J. A.; Blaylock, D. W.; Witt, P. M.; Turner, R. M.; Heider, P. L.; Harji, B. H.; Browne, D. L.; Ley, S. V. Reconfiguration of a Continuous Flow Platform for Extended Operation: Application to a Cryogenic Fluorine-Directed ortho-Lithiation Reaction. Org. Process Res. Dev. 2014, 18, 12211228. (i) Brodmann, T.; Koos, P.; Metzger, A.; Knochel, P.; Ley, S. V. 
Continuous Preparation of Arylmagnesium Reagents in Flow with Inline IR Monitoring. Org. Process Res. Dev. 2012, 16, 1102-1113.

(8) For the use of ultrasound to overcome microreactor clogging, see: (a) Chen, Y.; Sabio, J. C.; Hartman, R. L. When Solids Stop Flow Chemistry in Commercial Tubing. J. Flow Chem. 2015, 5, 166-171.

(b) Hartman, R. L. Managing Solids in Microreactors for the Upstream Continuous Processing of Fine Chemicals. Org. Process Res. Dev. 2012, 16, 870-887. (c) Kuhn, S.; Noel, T.; Gu, L.; Heider, P. L.; Jensen, K. F. A Teflon Microreactor with Integrated Piezoelectric Actuator to Handle Solid Forming Reactions. Lab Chip 2011, 11, 2488-2492. (d) Noel, T.; Naber, J. R.; Hartman, R. L.; McMullen, J. P.; Jensen, K. F.; Buchwald, S. L. Palladium-Catalyzed Amination Reactions in Flow: Overcoming the Challenges of Clogging via Acoustic Irradiation. Chem. Sci. 2011, 2, 287-290.

(9) Nagaki, A.; Yoshida, J.-i. Preparation and Use of Organolithium and Organomagnesium Species in Flow. Top. Organomet. Chem. 2015, 57, 137-176.

(10) For the benefits of microreactor technology for photochemistry, see: (a) Noel, T. A Personal Perspective on the Future of Flow Photochemistry. J. Flow Chem. 2017, 7, 87-93. (b) Cambié, D.; Bottecchia, C.; Straathof, N. J. W.; Hessel, V.; Noël, T. Applications of Continuous-Flow Photochemistry in Organic Synthesis, Material Science, and Water Treatment. Chem. Rev. 2016, 116, 10276-10341. (c) Loubiere, K.; Oelgemoeller, M.; Aillet, T.; Dechy-Cabaret, O.; Prat, L. Continuous-Flow Photochemistry: A Need for Chemical Engineering. Chem. Eng. Process. 2016, 104, 120-132. (d) Mizuno, K.; Nishiyama, Y.; Ogaki, T.; Terao, K.; Ikeda, H.; Kakiuchi, K. Utilization of Microflow Reactors to Carry Out Synthetically Useful Organic Photochemical Reactions. J. Photochem. Photobiol., C 2016, 29, 107-147.

(11) Attempts to connect the two steps in one flow protocol failed. Apparently impurities from the Grignard reaction negatively affect the photocatalytic step. Even using inline purification steps, such as microfluidic extractions, did not solve this issue.

(12) O'Hara, F.; Blackmond, D. G.; Baran, P. S. Radical-Based Regioselective C-H Functionalization of Electron-Deficient Heteroarenes: Scope, Tunability, and Predictability. J. Am. Chem. Soc. 2013, 135, 12122-12134.

(13) Wei, X.-J.; Boon, W.; Hessel, V.; Noel, T. Visible-Light Photocatalytic Decarboxylation of $\alpha, \beta$-Unsaturated Carboxylic Acids: Facile Access to Stereoselective Difluoromethylated Styrenes in Batch and Flow. ACS Catal. 2017, 7, 7136-7140.

(14) (a) Straathof, N. J. W.; Su, Y.; Hessel, V.; Noël, T. Accelerated Gas-Liquid Visible Light Photoredox Catalysis with Continuous-Flow Photochemical Microreactors. Nat. Protoc. 2016, 11, 10-21. (b) Bottecchia, C.; Wei, X.-J.; Kuijpers, K. P. L.; Hessel, V.; Noël, T. Visible Light-Iinduced Trifluoromethylation and Perfluoroalkylation of Cysteine Residues in Batch and Continuous Flow. J. Org. Chem. 2016, 81, 7301-7307.

(15) Yang, H.; Huo, N.; Yang, P.; Pei, H.; Lv, H.; Zhang, X. Rhodium Catalyzed Asymmetric Hydrogenation of 2-Pyridine Ketones. Org. Lett. 2015, 17, 4144-4147. 